\title{
Comprehensive classification of the plant non-specific lipid transfer protein superfamily towards its Sequence - Structure - Function analysis.
}

\author{
Cecile Fleury $^{1}$, Jerome Gracy ${ }^{2}$, Marie-Francoise Gautier ${ }^{1}$, Jean-Luc Pons ${ }^{2}$, Jean-Francois Dufayard ${ }^{3}$, Gilles \\ Labesse $^{2}$, Manuel Ruiz ${ }^{3}$, Frederic de Lamotte ${ }^{\text {Corresp. } 1}$ \\ 1 UMR AGAP, INRA, F-34060 Montpellier, France \\ 2 CBS, CNRS Univ Montpellier INSERM, Montpellier, France \\ 3 UMR AGAP, CIRAD, F-34398 Montpellier, France \\ Corresponding Author: Frederic de Lamotte \\ Email address: frederic.de-lamotte@inra.fr
}

Background. Non-specific Lipid Transfer Proteins (nsLTPs) are widely distributed in the plant kingdom and constitute a superfamily of related proteins. More than 800 different sequences have been characterized so far, but their biological functions remain unclear. It has been clear for years that they present a certain interest for agronomic and nutritional issues. Deciphering their functions means collecting and analyzing a variety of data from gene sequence to protein structure, from cellular localization to the physiological role. As a huge and growing number of new protein sequences are available nowadays, extracting meaningful knowledge from sequence-structure-function relationships calls for the development of new tools and approaches. As nsLTPs show high evolutionary divergence, but a conserved common right-handed superhelix structural fold, and as they are involved in a large number of key roles in plant development and defense, they are a stimulating case study for validating such an approach.

Methods. In this study, we comprehensively investigated 797 nsLTP protein sequences, including a phylogenetic analysis on canonical protein sequences, three-dimensional (3D) structure modeling and functional annotation using several well-established bioinformatics programs. Additionally, two integrative methodologies using original tools were developed. The first was a new method for the detection of i) conserved amino acid residues involved in structure stabilization and ii) residues potentially involved in ligand interaction. The second was a structure-function classification based on the Evolutionary Trace Display method using a new tree visualization interface. We also present a new tool for visualizing phylogenetic trees.

Results. Following this new protocol, an updated classification of the nSLTP superfamily was established and a new functional hypothesis for key residues is suggested. Lastly, this work allows a better representation of the diversity of plant nsLTPs in terms of sequence, structure, and function. 


\section{Comprehensive classification of the plant non-specific lipid transfer protein superfamily towards its Sequence - Structure - Function analysis}

Cécile Fleury ${ }^{1}$, Jérôme Gracy ${ }^{3}$, Marie-Françoise Gautier ${ }^{1}$, Jean-Luc Pons ${ }^{3}$, Jean-François Dufayard $^{2}$, Gilles Labesse ${ }^{3}$, Manuel Ruiz ${ }^{2}$, Frédéric de Lamotte ${ }^{1}$

${ }^{1}$ INRA, UMR AGAP, F-34060 Montpellier, France

${ }^{2}$ CIRAD, UMR AGAP, F-34398, Montpellier

${ }^{3}$ CBS CNRS Univ. Montpellier- INSERM, Montpellier, F-34090, France

Corresponding Author:

Frédéric de Lamotte ${ }^{1}$

Avenue Agropolis - TA A-108/03 - Montpellier - F-34398 Cedex 5 - France

Email address: frederic.de-lamotte@inra.fr

\section{ABSTRACT}

Background. Non-specific Lipid Transfer Proteins (nsLTPs) are widely distributed in the plant kingdom and constitute a superfamily of related proteins. More than 800 different sequences have been characterized so far, but their biological functions remain unclear. It has been clear for years that they present a certain interest for agronomic and nutritional issues. Deciphering their functions means collecting and analyzing a variety of data from gene sequence to protein structure, from cellular localization to the physiological role. As a huge and growing number of new protein sequences are available nowadays, extracting meaningful knowledge from sequence-structure-function relationships calls for the development of new tools and approaches. As nsLTPs show high evolutionary divergence, but a conserved common right handed superhelix structural fold, and as they are involved in a large number of key roles in plant development and defense, they are a stimulating case study for validating such an approach. Methods. In this study we comprehensively investigated 797 nsLTP protein sequences, including a phylogenetic analysis on canonical protein sequences, three-dimensional (3D) structure modelling and functional annotation using several well-established bioinformatics programs. Additionally, two integrative methodologies using original tools were developed. The first was a new method for the detection of i) conserved amino acid residues involved in structure stabilization and ii) residues potentially involved in ligand interaction. The second was a structure-function classification based on the Evolutionary Trace Display method using a new tree visualization interface. We also present a new tool for visualizing phylogenetic trees. Results. Following this new protocol, an updated classification of the nsLTP superfamily was established and a new functional hypothesis for key residues is suggested. 
Lastly, this work allows a better representation of the diversity of plant nsLTPs in terms of sequence, structure and function.

\section{INTRODUCTION}

Since the work of Kader (Kader et al., 1984; Kader, 1996), numerous proteins capable of transferring lipids have been annotated as non-specific lipid transfer proteins (nsLTPs). Their primary sequences are characterized by a conserved 8-Cysteine Motif (8CM) (C-Xn-C-Xn-CC$\mathrm{Xn}-\mathrm{CXC}-\mathrm{Xn}-\mathrm{C}-\mathrm{Xn}-\mathrm{C}$ ), which plays an important role in their structural scaffold (José-Estanyol et al., 2004). Based on their molecular masses, plant nsLTPs were first separated into two types: type I $(9 \mathrm{kDa})$ and type II $(7 \mathrm{kDa})$, which were distinct both in terms of primary sequence identity and the disulfide bond pattern (Douliez et al., 2001).

Plant nsLTPs are ubiquitous proteins encoded by multigene families, as reported in different phylogenetic studies. However, these studies involve a limited number of sequences and/or species: fifteen nsLTPs identified in Arabidopsis (Arondel et al., 2000), restricted to Poaceae (Jang et al., 2007) or Solanaceae (Liu et al., 2010). Around 200 nsLTPs have been identified in wheat, rice and Arabidopsis genomes and classified into nine different types according to sequence similarity (Boutrot et al., 2008). More extensive studies including ancestral plants indicate that nsLTPs are also present in liverworts, mosses and ferns, but not present in algae (Edstam et al., 2011; Wang et al., 2012).

- From a structural point of view, the nsLTP family belongs to the all-alpha class in the SCOP database (Murzin et al., 1995), as these small proteins contain four or five helices organized in a right-handed superhelix. To date, only 30 three-dimensional redundant structures corresponding to 8 different proteins have been experimentally determined. According to SCOP, the protein fold called "Bifunctional inhibitor/lipid-transfer protein/seed storage $2 \mathrm{~S}$ albumin" is found in at least six distinct plant nsLTPs for which the 3D structure has been solved (from five species Triticum aestivum, Hordeum vulgare, Zea mays, Oryza sativa and Triticum turgidum), and one soybean hydrophobic protein. In the RCSB Protein Database (Berman et al., 2000) we listed four more plant nsLTP 3D structures (from Nicotiana tabacum, Phaseolus aureus, Prunus persica and Arabidopsis thaliana). According to the CATH database (Orengo et al., 1997), nsLTPs belong to the "Mainly alpha" class. They display the "Orthogonal Bundle" architecture and the "Hydrophobic Seed Protein" topology. At this level, only one homologous superfamily called "Plant lipid-transfer and hydrophobic proteins" can be found. The superfamily appears to contain ten distinct protein sequences, lacking the $A$. thaliana nsLTP, but including the soybean hydrophobic protein found in the SCOP database. Of the known nsLTP 3D structures, only Boutrot's type I, II and IV are represented. An interesting point to be noted is that two different cysteine pairing patterns have been observed (which correspond to a single cysteine switch between two disulfide bridges): C1-C6 and C5-C8 in type I structures; C1-C5 and C6-C8 in type II and IV structures. However, C2-C7 and C3-C4 bridges are common to all known nsLTP structures and the overall fold is conserved among the whole family.

- From a functional point of view, plant nsLTPs are classified into different families depending on the scope of interest and their properties (Liu et al. 2015). Plant nsLTPs belong to the 
Prolamin superfamily (AF050), which includes the largest number of allergens (Radauer et al., 2008). Indeed, several nsLTPs from fruits of the Rosaceae family, nuts or different vegetables are food allergens, with fruit nsLTPs being mainly localized in the peel (Salcedo et al., 2007). Plant nsLTPs are members of the pathogenesis-related proteins and compose the PR14 family (van Loon et al., 2006). Their role in plant defense mechanisms has been shown by the induction of nsLtp gene expression following pathogen infections, overexpression in transgenic plants, or their antimicrobial properties (Molina \& García-Olmedo, 1993; Cammue et al., 1995; Li et al., 2003; Girault et al., 2008; Sun et al., 2008). A role in plant defense signaling pathways has also been suggested for an Arabidopsis type IV nsLTP, which needs to form a complex with glycerol3-phosphate for its translocation and induction of systemic acquired resistance (Maldonado et al., 2002; Chanda et al., 2011). One wheat nsLTP competes with a fungal cryptogein receptor in tobacco plasma membranes and, when the LTP is complexed with lipids, its interaction with the membrane and its defense activity are enhanced (Buhot et al., 2001; Buhot et al., 2004). In wheat, nsLtp genes display a complex expression pattern during the development of the seed (Boutrot et al., 2005). NsLTPs may also be involved in plant defense mechanisms through their participation in cuticle synthesis (Debono et al., 2009). This function is supported by their extracellular localization (Thoma et al., 1993; Pyee et al., 1994), the expression of different nsLtp genes in leaf epidermis (Sterk et al., 1991; Pyee \& Kolattukudy, 1995; Clark \& Bohnert, 1999), a positive correlation between $n s L t p$ gene expression and cuticular wax deposition (Cameron et al., 2006), and their ability to bind cutin monomers (i.e. hydroxylated fatty acids) (Douliez et al., 2001). In addition, nsLtp gene transcripts are abundant or specifically present in trichomes and one tobacco nsLTP seems to be required for lipid secretion from glandular trichomes indicating that nsLTPs may play a role either in the secretion of essential oils or in defense mechanism (Lange et al., 2000; Aziz et al., 2005; Choi et al., 2012). Several nsLtp genes are up or down-regulated by application of different abiotic stresses including low temperature, drought, salinity and wounding (Wang et al., 2012; Treviño \& O'Connell, 1998; Gaudet et al., 2003; Maghuly et al., 2009). A cabbage nsLTP isolated from leaves stabilizes thylakoid membranes during freezing (Sror et al., 2003). Transgenic orchids transformed with a rice nsLTP exhibit an enhanced tolerance to cold stress (Qin et al., 2011).

Function in male reproductive tissues has also been shown for a lily nsLTP involved in pollen tube adhesion (Mollet et al., 2000; Park et al., 2000) and the Arabidopsis LTP5 implicated in pollen tube guidance in the pistil (Chae et al., 2009; Chae \& Lord, 2011). A tobacco nsLTP that accumulates in pistils has been shown to be involved in cell wall loosening, and this activity relies on the hydrophobic cavity of the protein (Nieuwland et al., 2005).

NsLTPs are possibly involved in a range of other biological processes, but their physiological functions are not clearly understood. An analysis of gain of function or defective plant mutants can address these issues (Maldonado et al., 2002; Chae et al., 2009). Site directed mutagenesis led to the identification of residues involved in their antifungal activity, lipid binding and lipid transfer (Ge et al., 2003; Cheng et al., 2008; Sawano et al., 2008). However, these approaches are time-consuming and have so far been limited to a small number of proteins. 
There is a lack of bioinformatic tools enabling investigations into such complex superfamilies of proteins. Current programs such as GeneSilico Metaserver (Kurowski \& Bujnicki, 2003) or MESSA (Cong \& Grishin, 2012) provide an overview of known information about protein sequences, structures and functions, but studying inner relationships on a large scale requires a knowledge visualization and classification tool that still needs to be developed.

As nsLTPs show high evolutionary divergence but a conserved common fold, and as they are involved in a large number of key roles in plant development and defense, the nsLTP superfamily constitutes a very interesting case study for validating such a method.

\section{MATERIALS \& METHODS}

\section{1/NsLTP sequences}

\section{Definition of the protein sequence set}

A first pool of plant nsLTPs sequences was retrieved from the UniProtKB (Swiss-Prot + TrEMBL) (http://www.uniprot.org), Phytozome (http://www.phytozome.net) and NCBI databases (http://www.ncbi.nlm.nih.gov), using either Blast or keyword queries ("Plant lipid transfer protein", "viridiplantae lipid transfer protein", "plant A9 protein", "A9 like protein", "tapetum specific protein", "tapetum specific", "anther specific protein”, "A9 Fill”). Original data obtained on the Theobroma cacao genome were also investigated (Argout et al., 2011). From this large pool of proteins, the plant nsLTP dataset was defined according to a new set of criteria: (i) sequences from 60 to 150 residues long, including signal peptide; (ii) containing strictly eight cysteine residues after removal of the signal peptide; (iii) cysteine residues distributed in the 8CM pattern (C-Xn-C-Xn-CC-Xn-CXC-Xn-C-Xn-C). We excluded multidomain proteins, i.e. the hybrid proline-rich and hybrid glycine-rich proteins in which the signal peptide is followed by a proline-rich or a glycine-rich domain of variable length (José-Estanyol et al., 2004). For each sequence, the signal peptide was detected and removed using SignalP 3.0 (Bendtsen et al., 2004). In all, including the wheat, rice and Arabidopsis sequences previously identified by Boutrot (Boutrot et al., 2008), 797 non-redundant mature amino acid sequences belonging to more than 120 plant species were kept for analysis.

\section{Sequence alignments and phylogenetic analysis}

In order to achieve the best alignment, the pool of 797 sequences was aligned using both the MAFFT (Katoh et al., 2002; Katoh \& Toh, 2010) and MUSCLE (Edgar, 2004) programs with respective parameters of 1.53 for gap opening, 0.123 for gap extension and BLOSUM62 matrix; maximum iteration 16 .

The two resulting multiple alignments were compared and conflicts between the two were highlighted. To discriminate between the two different cysteine patterns suggested (see Results section), a restricted analysis was carried out using only the $10 \mathrm{nsLTPs}$ for which at least one structure had previously been experimentally determined. Two new 10 -sequence alignments 
were calculated, one by MUSCLE and one by MAFFT. Using the ViTo program (Catherinot \& Labesse, 2004), each alignment was projected on type I, II and IV nsLTP 3D structures, and the spatial distance of equivalent cysteine residues was evaluated. The alignment that minimized these distances was selected as the best one.

Based on the best alignment, a phylogenetic tree was calculated using PhyML (Guindon et al., 2010). Lastly, the tree was reconciled with the overall species tree using the Rap-Green program (Dufayard et al., 2005).

\section{$\underline{2 / \text { NsLTP three-dimensional structures }}$}

\section{Three-dimensional structure modeling}

For 10 out of the 797 nsLTP dataset, one or more experimentally determined 3D structures were available and downloaded from the Protein Data Bank (http:/www.rcsb.org/pdb). Theoretical structures were calculated for the other 787 proteins using the @ tome2 suite of programs to perform homology modeling (Pons \& Labesse, 2009) (http://atome.cbs.cnrs.fr). The quality of each final structure model was evaluated using Qmean (Benkert et al., 2008). Structures with low quality (i.e. for which the cysteine scaffold could not be fully modeled) were discarded from further analysis (see Table 1).

\section{Structural classification}

All the remaining good-quality theoretical structures, together with the 10 experimental structures composed the 3D structure pool of the study. Except for the cysteine pattern analysis by ViTo, this structural pool was used in all further structural analysis.

The structures were compared to each other in a sequence-independent manner, using the similarity matching method of the MAMMOTH program (Ortiz et al., 2002). The RMSD was calculated for each pair of structures, using the superposition between matched pairs that resulted in the lowest RMSD value. This superposition was computed using the Kabsch rotation matrix (Kabsch, 1976; Kabsch, 1978) implemented in the MaxCluster program (Herbert, http://www.sbg.bio.ic.ac.uk/maxcluster, unpublished). We used the RMSD score matrix calculated by MaxCluster as input for the FastME program (Desper \& Gascuel, 2002) to calculate a structural distance tree.

\section{3/ NsLTP functional annotation}

Extensive bibliographic work was carried out to collect and classify functional information available in the literature about the nsLTPs of the dataset. Gene Ontology (GO), Plant Ontology (PO) and Trait Ontology (TO) terms were collected from the Gramene Ontologies Database (http://www.gramene.org/plant_ontology) and organized in a dedicated database, together with the bibliographic references when available. The database was also enriched with additional information, such as methods used for gene expression studies (northern, RT-PCR or microarray data, in situ hybridization), protein purification, in vitro or in planta antifungal and antibacterial 
activity, lipid binding or transport (fluorescence binding assay or in vitro lipid transfer). Information about tissues and organs used in cDNA libraries was collected from the NCBI databases (http://www.ncbi.nlm.nih.gov).

\section{4/Integrative method 1: sequence $->$ structure $->$ function}

This method seeks to identify common ligand binding properties in nsLTPs clustered by sequence similarity.

\section{Sequence consensus for each nsLTP type}

797 nsLTP sequences were clustered by type on the basis of regular expressions derived from the consensus motifs described in (Boutrot et al., 2008). Each type subfamily was then aligned individually and the resulting sequence profiles were globally aligned using MUSCLE. For each type subfamily, the most frequent amino acids were selected at each alignment position to build the consensus sequence. A consensus amino acid was replaced by a gap if more than half of the sequences were aligned with a deletion at the considered position.

\section{NsLTP sequence-structure analysis using Frequently Aligned Symbol Tree (FAST)}

An original tool was designed to highlight conserved amino acid positions specific to each nsLTP phylogenetic type, and which might be decisive for their function. The algorithm relied on a statistical analysis of each alignment row, after the sequences had been clustered according to their phylogenetic distances.

For each type subfamily, the most frequent amino acids were selected at each alignment position to build the consensus sequence. A consensus amino acid was replaced by a gap if more than half of the sequences were aligned with a deletion at the considered position. We then calculated the amino acid conservations and specificities over each column of the multiple sequence alignment to delineate the functionally important residues in each nsLTP subfamily. This statistical analysis is explained in the appendix file.

In order to visualize the conserved and divergent regions of the sequences, different color ranges were assigned to the nsLTP phylogenetic subfamilies. Conserved amino acid positions along the whole alignment (CCP: Conserved Core Positions) are represented in grey/black, while specifically conserved positions among proteins of the same subfamily (SDP: Specificity

Determining Positions) are represented in saturated colors corresponding to the family ones. The tool enabled scrolling down of the alignment to easily identify both types of conserved positions and two distant parts of the alignment could be displayed together to compare distant phylogenetic subfamilies.

Contacts with ligands, solvent accessibility and other parameters could also be displayed above the alignment. Using the Jmol interface, conserved amino acid residues could be projected on nsLTP representative 3D structures, so that the potential role of each position could be interpreted geometrically. 


\section{5/ Integrative method 2: function $->$ structure $->$ sequence}

Structural Trace Display is a method, based on Evolutionary Trace Display (ETD, Erdin et al.,2010), that seeks to identify common structural (1D, 3D) properties in nsLTPs sharing similar functions.

\section{Clustering of the structure tree}

As in a phylogenetic tree, nsLTPs in the structure tree were clustered according to their similarity. In the case of this particular tree, the similarity between nsLTPs was measured by a spatial distance in angströms (see paragraph 2/ NsLTP three-dimensional structures / Structural classification). Decreasing distance cutoffs ranged from $11.5 \AA$ (one cluster containing all nsLTP structures) to $0.5 \AA$. Each cutoff application caused a division of the tree into one or more subtrees that contained leaves (i.e. nsLTP structures) whose structural proximity altogether (represented by the pairwise RMSDs) was up to the value of the applied cutoff.

\section{InTreeGreat: an integrative tree visualization tool}

We developed an integrative tree visualization tool called InTreeGreat in order to display the whole or some parts of either sequence or structure distance trees.

InTreeGreat was implemented using PHP and Javascript, in order to generate and manipulate an SVG graphical object.

The main objective of this tool is to graphically highlight correlations between 3D structures, evolution, functional annotations or any available heterogeneous data. In the context of this study, the interface was able to retrieve information from the nsLTP database to annotate the tree.

InTreeGreat includes functionalities such as tree coloration, fading, and collapsing. Heterogeneous data related to sequences (e.g. annotations, nsLTP classification) can be displayed in colored boxes, aligned to the tree.

\section{Cluster Selection}

Using InTreeGreat to investigate our annotated structure tree, we looked for clusters of nsLTPs sharing the same kind of functional annotations. We focused our attention on one interesting functional role highlighted in several nsLTPs: the implication in plant defense mechanisms against pathogens (bacteria and/or fungus). In order to highlight structure-function relationships, we studied three groups of nsLTPs (see Results section for details): (i) the so-called "defense cluster" (43 proteins, distance cutoff $=1.5 \AA$ ); (ii) the cluster containing all type I fold proteins (402 proteins, distance cutoff = $3 \AA$ ); (iii) a group manually composed of all type I fold nsLTPs for which a functional role in defense and/or resistance against pathogens had been reported in the literature (28 proteins).

Within each of these 3 clusters, the protein structure showing the shortest RMS distance from all the others was selected as the representative structure of the cluster for the structural trace calculation. 


\section{Structural Trace calculation}

A structure-based sequence alignment was carried out on the nsLTP structures by Mustang software (Konagurthu et al., 2006).

For each previously selected structural cluster, the corresponding set of protein sequences was extracted from the multiple structural alignment of the nsLTPs. The Evolutionary Trace (ET) method (Lichtarge et al., 1996) was applied: the partial multiple sequence alignment was submitted as input for the ETC program (locally installed, http://mammoth.bcm.tmc.edu/ETserver.html) together with the representative structure of the cluster (selected as described in the previous paragraph).

The "evolutionary" traces based on the structural alignments corresponding to the three nsLTP clusters were then compared to each other. To that end, the $30 \%$ top-ranked residues of the defense cluster trace were considered as constitutive of the reference trace (i.e. 27 most conserved amino acid residues) and their ranking and scores in the two other traces were analyzed. The results were compiled in a table and graphically visualized using PyMOL (http://www.pymol.org/).

\section{RESULTS}

\section{1/ NsLTP sequences analysis}

\section{NsLTP dataset}

Over the last four decades numerous proteins, whose ability to transfer lipids has not always been demonstrated, have been annotated as nsLTPs on the basis of sequence homology. In order to understand more clearly the functional characteristics and the inner variability of this family, we focused the study on the monodomain proteins, which present the strict and only nsLTP domain, i.e. the eight-cysteine residues arranged in four disulfide bridges. In total, including the wheat, rice and Arabidopsis sequences previously identified (Boutrot et al., 2008), together with sequences from the UniProt (Swiss-Prot/TrEMBL), NCBI and Phytozome databases, 797 nonredundant mature nsLTP sequences belonging to more than 120 plant species were kept for analysis. This first step allowed the selection of a relevant set of proteins covering variability in the nsLTP family. The number of sequences (798) was also large enough to challenge any analysis method we used during this study.

\section{Sequence alignment and Cysteine pattern}

The alignment of all non-redundant protein sequences for which the 3D structure was experimentally determined (10 sequences) was carried out twice, using the MUSCLE program on the one hand, and the MAFFT program on the other hand. The resulting alignments obtained with standard settings are shown on Figures 1A1 and 1B1.

In both cases, cysteine residues of the $8 \mathrm{CM}$ aligned quite well among the three represented types of nsLTPs (types I, II and IV), except for the Cys5-X-Cys6 (CXC) pattern region (where X 
stands for any amino acid residue). MUSCLE did align type I Cys5 with types II and IV Cys5', as well as type I Cys6 with types II and IV Cys6' (Figure 1A1), just as previous studies typically showed (Liu et al., 2010; Siverstein et al., 2007). However, in the alignment carried out by MAFFT (Figure 1B1), Cys5 of type I nsLTPs was equivalent to Cys6' of type II and IV nsLTPs, and not to the corresponding Cys5'.

While looking at the structures using ViTO, the small shift suggested by MAFFT alignment demonstrated better spatial correspondence between type I Cys5 and type II Cys6' (Figure 1B2). The superposition of the 3D structures of types I and II nsLTPs showed that Cys5 and Cys6 of type I nsLTPs could not be superimposed on Cys5' and Cys6', respectively, of type II nsLTPs (Figure 1A2), whereas Cys5 of type I nsLTPs could be superimposed on Cys6' of type II nsLTPs (Figure 1B2). Note that the value of the RMSD between C-alpha of the superimposed Cys residues calculated for the two alignment options dropped from 7.32 to 2.15 with the second alignment, as shown by Figures 1A2 and 1B2. Furthermore, with the alignment we suggest, type I hydrophylic X residue was exposed to the solvent, whereas type II apolar X residue was orientated toward the core of the protein, increasing the stability of the proteins.

This compound approach allowed us to sort the 798 sequences unambiguously into two main families.

\section{NsLTP sequence classification}

Our dataset was mainly composed of nsLTPs from angiosperm species (19 monocotyledonous species and 83 eudicotyledonous species) plus five gymnosperm species (35 sequences), one lycophyte species (34 sequences) and two bryophyte species (17 sequences). The monocot sequences were mainly represented by Poales nsLTPs (256 out of 270 sequences) whereas Rosid nsLTPs were the most abundant (364 out of 436 sequences) within eudicots.

The phylogenetic analysis showed that the pool of proteins clustered into nine different types, all highly supported (branch support $>0.84$ ). This result mostly confirmed Boutrot's classification, defined on A. thaliana, T. aestivum and O. sativa nsLTP sequences, in nine types (Boutrot et al., 2008). The main differences were the identification of a new group (named type XI), including 23 sequences, and that Boutrot's type VII nsLTPs disappeared from our dataset. Indeed, the latter did not satisfy the $8 \mathrm{CM}$ criteria as they have only seven cysteine residues in their sequences. For the same reason, Wang's A, B, C and D types (Wang et al., 2012) were not represented in our classification.

Type I nsLTPs formed a well-supported monophyletic group (branch support of 0.879) and predominated over the other types, as they accounted for more than half of our dataset (417 out of 797 sequences). This was also observed by Wang (Wang et al., 2012) with a set of 595 nsLTPs. Conversely, in Solanaceae, the most abundant nsLTPs belong to a type referred to as type $\mathrm{X}$ by Wang (70 out of 135 sequences) and which seems specific to that plant family (Liu et al., 2010) but was not present in our dataset. To avoid any confusion, we did not used type $\mathrm{X}$ denomination in this work. Type II nsLTPs were the second most abundant type (126 sequences) 
followed by type V (70 sequences) and type VI (60 sequences). Type IX (12 sequences) was mainly composed of Physcomitrella patens nsLTPs and type XI (23 sequences) was mainly composed of nsLTPs from eudicot species. Twelve nsLTPs were not included in any of the identified types: these were mainly P. patens (6 sequences) and S. moellendorfii (4 sequences) proteins (Figure 2).

Type XI were grouped in a cluster of 23 sequences in the phylogenetic tree, fairly well supported by a branch of 0.879 aLRT SH-like score. Type XI appeared between type I and the other types, but even though type XI and I were grouped together in the tree, it remained unclear which of the 3 groups (type I, type XI, and other types) diverged first.

All nsLTP types were represented in eudicots while types IX, X (in Wang's nomenclature) and XI were not identified in monocot species. Within the lycophyte and bryophyte species, no type II, III, IV nor VIII nsLTPs were identified. In the same way, no type III, VIII, IX or XI were identified within gymnosperm species. Ten out of the 16 moss $P$. patens nsLTPs were type IX, the other 6 remained un-typed, and the only liverwort Marchantia polymorpha nsLTP was a type VI. The 34 S. moellendorfii sequences were mainly types V and VI (15 and 7, respectively) and seven nsLTPs belonged to the new type XI. The $P$. patens and $S$. moellendorfii nsLTPs formed independent branches or were located at the same branch as type $\mathrm{V}$ in Wang's phylogenetic tree (Wang et al., 2012) and were included in type D in Edstam's classification (Edstam et al., 2011). However, Edstam's type D included rice and Arabidopsis type IV, V and VI nsLTPs. Edstam's type G nsLTPs, which corresponded to GPI-anchored LTPs and types J and K, which did not fit our molecular mass criteria or contain more than one $8 \mathrm{CM}$ motif were not included in our dataset.

According to Yi and coworkers (Yi et al., 2009), Allium nsLTPs may constitute a novel type of nsLTPs harboring a C-terminal pro-peptide localized in endomembrane compartments. In the prolamin superfamily tree of Radauer and Breiteneder (Radauer \& Breiteneder, 2007), the Allium cepa nsLTP (192_ALLCE) is closed but not included in the type I nsLTPs. In our phylogenetic tree, the three nsLTPs from Allium species were classified as type I. The 501_MEDTR medicago nsLTP was suggested to belong to a new nsLTP subfamily involved in lipid signaling (Pii et al., 2010) like Arabidopsis DIR1 (151_typeIV_ARATH). In our phylogenetic tree, both proteins were identified as type IV nsLTPs.

The Theobroma cacao genome contains at least 46 nsLtp genes distributed across the ten chromosomes. Several T. cacao nsLtp genes are organized in clusters, as observed in the rice, Arabidopsis and sorghum genomes (Boutrot et al., 2008; Wang et al., 2012). Apart from nine sequences that were classified in the new type XI, all other $T$. cacao nsLTPs were classified within the previously identified types and belonged mainly to type I (14 sequences), type VI ( 7 sequences) and type $\mathrm{V}$ (6 sequences). 
It is worth noting that all the nsLTPs identified as allergens (IgE binding) were type I, except one type II nsLTP (545_BRACM). The 501_MEDTR nsLTP was also suggested to play a role in the root nodulation process (Pii et al., 2009; Pii et al., 2013). Lipid signaling (lysophosphatidylcholine) has been reported to be involved in symbiosis (Bucher et al., 2009). This analysis was the most extensive so far and confirmed most of Boutrot's classification, but complements it due to a larger dataset and a more detailed phylogeny analysis.

\section{$\underline{\text { 2/ NsLTP structure analysis }}$}

\section{NsLTP structure modeling}

Given the nsLTP fold conservation and the quality of the available experimental structures, reliable models could be obtained for all nsLTPs using the comparative modeling method, although the sequence identity observed among all nsLTP sequences was only in the range of $25 \%$.

Models deduced by fold-recognition using the @TOME-2 server displayed overall good quality, as shown in Table 1 summarizing the Qmean scores. For 96\% of the models, Qmean scores were above 0.4 , and $57 \%$ of the models obtained scores ranging from 0.5 to 0.9 ., corresponding to scores for high-resolution proteins.

For 121 theoretical structures, the polypeptide chain could not be fully built and the resulting models were lacking at least one of the 8 cysteine residues. Such models were discarded and only the complementary pool of 677 structures was kept for further analysis.

All the structural alignments and three-dimensional models are available at:

http://atome.cbs.cnrs.fr/AT2B/SERVER/LTP.html

\section{NsLTP sequence - structure relationships}

In order to challenge the structure - function relationship analysis on such a big set, we decided to develop a new tool called FAST, which builds consensus sequences for each family, and highlights the sequence conservation and specificities on the alignment and the associated 3D structures.

Figure 3 shows the consensus sequence alignment for all nsLTP types. The pool of 797 sequences was clustered by type on the basis of regular expressions derived from the consensus motifs described by Boutrot and coworkers (Boutrot et al., 2008). Each type subfamily was then aligned individually and the resulting sequence profiles were globally aligned using MUSCLE.

Many residues specifically conserved in type I nsLTP1 corresponded to important folding differences between type I nsLTPs on the one side and all other LTP types on the other side. In the following sections, we list type I nsLTP-specific residues whose differential conservation was supported by structural or experimental data. 
First, Gly37, which was specifically conserved in type I nsLTPs, allowed very tight contact of helix 1 and helix 2, which were connected by the disulfide bridge Cys17-Cys34. The closest backbone distance between position 13 of helix 1 and position 37 of helix 2 was $3.34 \AA$ in a type I nsLTP structure (PDB code 1mid) while it was $6.45 \AA$ in a type II nsLTP structure (PDB code 1tuk). These increased helix distances closed the ligand tunnel, which was opened in type I nsLTPs between helix 1 and helix 3, and created two distinct cavities separated by a septum in type II nsLTPs (Hoh et al., 2005). Larger distances between helix 1 and helix 2 were predicted in all nsLTP sequences where Gly37 was mutated into larger residues (i.e. all types but I and XI) and should cause major rearrangement of the ligand cavity entrance on this side of the proteins. Arginine and lysine residues at position 51 and bulky hydrophobic residues at positions 87 and 89 were two other conserved specificities among type I nsLTPs. The side chains at position 51 had type I-specific polar interactions with the ligand at the cavity entrance near the C-terminal loop, which were not found in other nsLTP types, as detailed later in Figure 4.

In addition, in type I nsLTPs, the 5th and 6th cysteine residues belonged to helix 3 and were bridged with the first and $8^{\text {th }}$ cysteines, respectively. These two-disulfide bridges tightened both sequence termini to the protein core. Conversely, in types II and IV nsLTPs, the 5th and 6th cysteines showed permuted bridging partners (to 8th and $1^{\text {st }}$ cysteines, respectively). The intermediate residue connecting the 5th and 6th cysteines was exposed to solvent in type I nsLTPs, while it was replaced by a bulky hydrophobic residue interacting with the ligand in the type II and IV nsLTP core at position 54 of the alignment. It was shown by site-directed mutagenesis that the replacement of this intermediate residue by an alanine residue perturbed folding, ligand binding and lipid transfer activity in type II nsLTPs (Cheng et al., 2008). In the light of these experiments, it is therefore interesting to note that alanine residues were frequent at position 54 in type I nsLTPs, while larger hydrophobic residues almost always occupied this buried position in other nsLTP types.

The mutation to alanine of the residue at position 63 was also shown experimentally to be destabilizing in type II nsLTPs (Cheng et al., 2008). This position was occupied by large hydrophobic residues in all nsLTPs but types I and V, where alanine residues were frequent, and type III, where it corresponded to a deletion of 12 consecutive residues.

Other residues specifically conserved in type I nsLTPs were helix N-capping Thr6 and Thr47, whose side chains formed stabilizing hydrogen bonds with the protein backbone, and Tyr20, which was the center of a conserved hydrophobic cluster with Pro30 and Leu/Ile79. The interaction of Tyr20 with Pro30 was experimentally confirmed by the large up field shift of

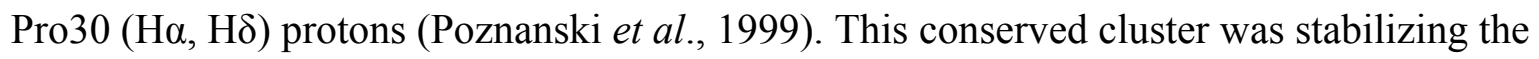
interface between helices 1 and 4, but did not participate in the ligand cavity. This particular helix interface was also observed in nsLTP types III, VI, VIII and XI. 
We then analyzed the atomic interactions observed between type I nsLTPs and their associated ligands in 19 PDB structures (supplementary data). Most contacts involved hydrophobic side chains of the type I nsLTP proteins and carbons of the ligands. Marginally, the most frequent polar contacts involved the side chains of conserved arginines at position 46 of the type I nsLTP alignment, lysines at position 54, aspartic acids at position 90, and various polar atoms of histidines, lysines and asparagines at position 37. It should be stressed that none of these polar interactions were shared by more than $31 \%$ of the protein-ligand complexes (fewer than $6 / 19$ PDB structures) although the least similar protein pair from the 19 structure set shared $67 \%$ sequence identities. This low level of polar contact conservation in homologous proteins with very similar sequences clearly indicated that no specific polar interactions anchored the proteinligand complexes in particular conformations. From this statistical analysis of protein-ligand polar contacts that did not exhibit a preferential cavity region for the interaction with the ligand polar heads, it could not be concluded that there was a preferred ligand orientation in the type I nsLTP tunnel. This observation was supported by recent protein-docking simulations and protein binding evaluations, which also concluded on a lack of preferred orientations of the ligand in the cavities of type I nsLTPs, and clear dominance of hydrophobic interactions in the protein-ligand interface (Pacios et al., 2012).

Lastly, positions 82 to 94 , which corresponded to the C-terminal loop, included some more residues specifically conserved in nsLTPs. This loop was much longer in type I nsLTPs than in other types, and had a major impact on the orientation of the ligand in the cavity, as shown in Figure 4.

\section{Conserved and specific residues in the nsLTP family}

The potential impact of variability within the nsLTP family on the tree dimensional structure of the proteins was further investigated. As shown in Figure 4, the ligand cavity opening near the CTerminal loop was very different when we compared the nsLTP structures of type I versus those of types 2 and 4 . The C-terminal loops connected the 4 helices to the 3 helices through the disulfide bridge between cysteine residues localized at alignment positions 95 and 55. Both helices 2 and 3 and the C-terminal loop were longer in type I than in types II and IV nsLTPs. In the type I nsLTPs, these elongations created a ligand cavity entrance along an axis perpendicular to the figure plane, while in types II and IV nsLTPs, the entrance was approximately parallel with the figure plane. Consequently, ligands would access the cavities on opposite sides of the Cterminal loop in type I versus types II and IV nsLTPs. Helix 2 and 3 were extended by an extra turn in type I nsLTPs comparatively to the structures of the other types. Moreover, the small space left in between helices 2 and 3 and the C-term loop was capped in types II and IV by bulky hydrophobic residues (Phe54 in 1tuk and Phe51 in 2rkn), while that position was occupied by a positively charged lysine or arginine in type I nsLTPs (red colored Arg51 in 1mid), whose side chain formed a hydrogen bond with the polar tail of the ligand. 
The structural differences observed between type I nsLTPs versus types II and IV can be generalized to other nsLTP types by looking at the alignment of consensus sequences in Figure 3. First, the extension of helices 2 and 3 in type I nsLTPs corresponded to a 6- to 8-residue insertion in the consensus sequence alignment, which differentiated type I from every other type of nsLTPs. Secondly, the C-terminal loop connecting the last two cysteine residues was, on average, 13 residues long in type I nsLTPs, while this loop was shortened to 6, 6, 7, 12, 9, 8, 6 and 9 residues long in types II, III, IV, V, VI, VIII, IX and XI, respectively. Lastly, the capping hydrophic residues at positions 54 and 51 of types II and IV nsLTPs were also observed in all the other nsLTP types. These conserved differences between type I and other types of nsLTP sequences indicated with high confidence that the global fold of type I LTP differed from the fold of the other nsLTP types and that the ligand cavity entries in type I nsLTPs were uniquely located.

The fold of type I nsLTPs will be hereafter referred to as "Type-1 fold" and the alternative fold of Types II to XI will be referred to as "Type-2 fold". (in other words: roman numeral I to XI correspond to phylogeny analysis while Arabic numeral 1 or 2 refer to structural analysis)

The preceding analysis of the evolutive conservations specific to type I nsLTPs revealed many residues whose role could be explained by local structural differences with the available types II and IV nsLTP structures. This comparative structure analysis confirmed the clear separation between type I and all the other nsLTP types initially observed in the phylogenetic tree inferred from a multiple sequence alignment of the 797 available proteins. The key residues were usually present in type I nsLTPs only and suggested that many structural differences observed when comparing type I versus types II and IV nsLTPs should also be observed versus other nsLTP types, particularly regarding ligand orientation and cavity entrances. This observation should guide the choice of templates when nsLTP types with unknown structures are modeled by homology.

\section{Structure classification}

In order to correlate the evolution of protein sequences and the impact on the corresponding structures, we produced a circular tree according to structural distances (Fig. 5). Whereas type I remained together in this second classification, other phylogenetic types were relatively scattered in the tree. A majority of type II nsLTPs remained together in this tree, as was also the case for type IV and type III, but no clear and reliable segregation between all non-type I nsLTPs could be made. Looking at the 3D structures allowed us to confirm the hypothesis that only two major structural types could be distinguished. They will be hereafter referred to as "Type-1 fold" and "Type-2 fold".

Several studies also showed that type I and type II nsLTPs differed through the characteristics of the residue standing between Cys5 and Cys6, being respectively hydrophilic in type I and apolar in type II proteins (Douliez et al., 2001; Marion et al., 2004). Based on the multiple sequence alignment of the 797 nsLTPs and observation of the nature of the central residue in the CXC 
pattern, together with the observations made in the preceding sequence-structure analysis, we suggest that types III, IV, V, VI, VIII, IX and XI nsLTP C5 and C6 residues will adopt the same spatial conformation as type II proteins, i.e. the so-called "Type-2 fold".

\section{NsLTP structure-function relationship}

Dealing with big datasets can be cumbersome and requires a very efficient interface. To address this challenge, we developed InTreeGreat, a Javascript/PHP interface, compatible with every standard web navigator. It is able to display and explore any tree and to deal with branch and leaf coloring, branch lengths, branch support (or any other branch labels), and can aggregate heterogeneous data (annotations, expression profiles, etc.). Figure 6 shows how InTreeGreat can be used to display phylogenic trees together with various types of annotations.

Among the annotated nsLTPs (433 out of 797), we focused on those that had been reported for their role in plant defense and/or resistance against pathogens (bacteria and/or fungi). To simplify, we shall hereafter refer to them as "defense nsLTPs" in the present discussion. By investigating structural similarities between the 31 identified defense nsLTPs in our annotated dataset, we attempted to identify key amino acid residues that may bestow their functional properties on these proteins.

Looking at the distribution of the defense nsLTPs in our structural classification (Figure 6) we observed that they were predominantly found in the type I part of the tree (28 proteins), with only 3 defense nsLTPs with a type II (85, 151, 501 - UniProtKB - P82900: Non-specific lipidtransfer protein 2G, Q8W453: Putative lipid-transfer protein DIR1, O24101: Lipid transfer protein). We therefore preferred to focus on the Type-1 fold nsLTPs and study the structural trace(s) inside this important subfamily of nsLTPs.

The cluster containing all Type- 1 fold defense nsLTPs corresponded to the whole type I part of the tree (402 members). The corresponding structural trace was calculated, but it could not be linked to the defense function, as the proportion of annotated nsLTPs with a defense function was too low ( 28 out of 402 , i.e. $7 \%$ ).

In order to obtain a meaningful trace of the potential defense function, we needed to select a cluster with a higher proportion of annotated defense nsLTPs. The best cluster we could find was a relatively small cluster (43 members) of proteins with a structural distance no greater than $1.5 \AA$ (i.e. 1.5 cut off), which contained $33 \%$ of the defense nsLTPs (i.e. 10 out of 31 proteins). This cluster will be referred to as "defense cluster" in the further discussion.

The structural trace of the defense cluster showed several differences in comparison with the structural trace of the Type-1 fold cluster (Table 2). Apart from the 8 Cys residues that were common to all nsLTPs, the $30 \%$ top ranked (i.e. 27 residues) most conserved residues were not the same, or did not come in the same order in both traces. According to the defense cluster 
trace, residue Asp at position 259 of the alignment (Asp45 in protein 525) was as strongly conserved as the 8 Cys residues. Residue Ile at position 402 (Ile80 in protein 525) was among the 4 best ranked residues after the 8 Cys residues and obtained a very low coverage, variability and rvET score. In terms of the ranking of these two (amino acid) residues in the Type-1 fold nsLTP trace, they appeared to occur much later in the ranking (20th and 21st rank, respectively) with much higher rvET scores and large variability in terms of the number and physico-chemical properties of the residues (Table 2). It can be suggested that these two residues were not critical for maintaining structure integrity, but could bestow functional specificity on the proteins classified in the defense cluster. In the trace obtained for the group composed by all the other Type- 1 fold defense nsLTPs, both residues Asp and Ile were among the 4 best ranked residues after the 8 Cys residues and also showed good coverage and rvEt scores (Table 2).

Three other residues located at positions 137, 154 and 266 of the structural alignment were differently conserved in the three clusters. Interestingly, these three positions showed good conservation ranking, but the variability of the three corresponding residues was notably higher in the Type-1 fold cluster. Indeed, in the defense cluster trace, position 137 was occupied either by a valine or by an alanine residue (Val7 in protein 525) and position 154 was occupied either by a leucine or by a valine residue (Leu11 in protein 525). Thus, both positions were occupied by hydrophobic residues in defense proteins, which was not always the case in Type- 1 fold proteins (Table 2). In the same way, position 266 was occupied either by an arginine or a lysine residue (both positively charged residues) (Lys46 in protein 525) in defense proteins, but allowed greater variability in terms of physicochemical properties in the other proteins harboring a Type- 1 fold. The fact that these three positions of the structural alignment belonged to the top $30 \%$ most conserved among all Type-1 fold nsLTPs suggested their importance in these proteins. However, because the variability at these three positions was very small among defense nsLTPs and because the physico-chemical property was strongly conserved, we suspected that residues located at positions 137, 154 and 266 of the structural alignment might bestow functional specificity, at least in the case of defense/resistance proteins.

Figure 7 shows the five residues highlighted in Table 2 in the 3D structural context of the representative protein of the defense cluster (protein 525). In this protein, conserved residues Asp and Ile were located at positions 45 and 80, respectively. The two small hydrophobic residues were Val7 and Leu11 and the positively charged residue was Lys46. All five key residues were located around the ligand cavity (Figure 7), which allowed either guidance or direct contact with the lipid. This observation was consistent with the suggested hypothesis.

NsLTP sequence-structure analyses using either FAST or STD revealed some key residues or key positions (in type I: Gly37, Arg/Lys51, bulky hydrophobic residues 87 and 89, Ala54, Thr6, 
Thr47, Tyr20, Pro30, Leu/Ile79, longer C-terminal loop; large hydrophobic residue 63 in types II, III, IV, VI, VIII, IX nsLTPs). The structural trace analysis highlighted other amino acid residues (in type I defense/resistance nsLTPs: Asp45, Ile80, Val/Ala7, Leu/Val11, Arg/Lys46). It is important to note that these two complementary analyses by FAST and STD were not meant to lead to the same kind of conclusions. Indeed, using sequence information projected on the 3D structure, the first method revealed nsLTP-type-specific amino acid residues that could be involved in structure stabilization and/or ligand interaction, given their structural context. The second method however considered a set of functionally close nsLTPs sharing a very similar structure and highlighted over-representatively conserved amino acid residues that might thus bestow functional specificity on these proteins. These two approaches took inverse directions in the path sequence - structure - function. The "sequence-to-function" method would lead to more precise conclusions if more data about the inner structural mechanisms of lipid binding were available (only a few structures of nsLTP-lipid complexes have been experimentally determined so far). The "function-to-sequence" method would give us a better overview of the range of nsLTP activities if the functional data were not so rare and heterogeneous.

However, we assumed that this combination of approaches i) allowed structure-sequence analysis for large multigene families, ii) could reveal structural patterns related to functions that were not revealed so far, as alignments would have been limited to primary sequences only and iii) allowed a comparison of groups composed of proteins with an evolutionary connection with groups displaying structural similarity.

\section{DISCUSSION}

A) We combined two powerful alignment algorithms (MAFFT and MUSCLE) together with a 3D projection of the impact of alignment on the structure of proteins (VITO). Real-time monitoring of the impact of gap positions and lengths on the resulting 3D model offered the possibility of discriminating between various alignment possibilities. This allowed us to provide definitive insight into the old debate about the CXC pattern and its implication for the structure of LTPs (Douliez et al. 2000). The resulting alignment allowed us to classify unambiguously all 798 sequences in main two nsLTP families.

B) The phylogenetic analysis was the most extensive to date, including 798 nsLTP sequences. This was a much more complete description than the previous one (195 sequences, Wang et al., 2012).

This phylogenetic analysis was conducted from a clearly defined dataset: sequences were selected using unambiguous parameters optimizing the quality of the output tree, also considering our 3D structural integration objective. Although GPI-Anchored LTP could have been included in this study, their incomplete homology with other LTPs and the lack of any experimental 3D structure, convinced us not to include them. Thanks to this choice, alignment quality was preserved, and a better-quality 3D structural model are used. This analysis allowed 
us to classify unambiguously all 798 sequences in the main two nsLTP families, complementing and reinforcing the former classification by Boutrot (Boutrot et al. 2008).

C) The production of more than 600 3D structural models and the collection of numerous functional annotations enabled progress to be made in the study of structure-function relationships of nsLTPs. The re-use of the ETD method in a close and adapted form (STD) led to the identification of amino acids involved in the functional specialization of some nsLTPs. STD allowed us to highlight amino acids specific to certain functions. One of the limiting points of this analysis remained the publication bias. Indeed, the annotations were not evenly distributed among available sequences, nor was it possible to distinguish between an unsearched function and a function not found. It seemed difficult to propose a solution to circumvent this bias (Douliez et al. 2000).

D) The structure tree clearly showed that all Type I ns-LTPs adopted the same folding (Type-1 fold), while all the other proteins adopted the second fold (Type-2 fold). This approach seemed very interesting but did not offer the same level of detail and the same analytical power as the phylogenetic approach. This was understandable, because phylogeny compares the different proteins with a much larger number of parameters (site-to-site mutation, classification of sites by mutation rate, use of refined distance matrix, etc.) while the structure tree only uses the RMSD of the structures taken 2 by 2 . While this innovative information was very interesting, it could potentially be improved if we had templates from each sub-family for the generation of molecular models (experimental structures are available for Type I, II and IV). Indeed, at this level of analysis, it is conceivable that models obtained from experimental structures for the other types (III, V, VI, VII, VIII, IX and XI) would provide improved models allowing the detection of other key residues.

\section{CONCLUSIONS}

Plant non-specific Lipid Transfer Proteins constitute a complex family of proteins whose biological functions are far from well understood. However, it has become clear for years that they are of increasing interest for agronomical and nutritional issues.

Experimental approaches are irreplaceable for accessing their inner functional mechanisms. However, such methods are expensive and time-consuming. Furthermore, they produce a large amount of heterogeneous data. For all these reasons, resorting to bioinformatics methods has long become necessary to organize and analyze existing data, and/or model and hypothesize new data.

This paper presented a new methodology based on the combination of either classical or original bioinformatics approaches, using various computational tools to extract information and suggest 
new hypotheses from a large pool of experimental data about the plant nsLTP superfamily of proteins.

In this paper, we:

1) Suggested a new definition of the nsLTP superfamily, with a set of criteria based on sequence length, sequence composition (e.g. Cys involved in SS bonds) and structure (monodomain).

2) Confirmed and enriched Boutrot's phylogenetic classification of plant nsLTP sequences.

3) Demonstrated the need for a small shift in the CXC alignment that reflected the existence of two main distinct nsLTP folds.

4) Calculated 666 good quality theoretical three-dimensional structures of nsLTPs.

5) Developed an original alignment tool to detect conserved and specific positions among the different phylogenetic types of nsLTPs.

6) Used the latter tool to reveal some key residues.

7) Suggested a new structure-based classification of the 676 nsLTP structures now available (10 experimental +666 theoretical), which that allow clustering by structural similarity.

8) Annotated all available information about the function.

9) Developed an original interface allowing quick visualization of several types of annotations on any phylogenetic tree.

10) Revealed, using structural trace analysis, potential specific amino acid residues involved in plant defense and/or resistance against pathogens

Our work was made more difficult by the problems of annotation bias for which we did not expect a practical solution. However, it seemed that some of our results could be improved if we had additional experimental structures for all types of nsLTP.

\section{ACKNOWLEDGEMENTS}

This work was supported by the CIRAD - UMR AGAP HPC Data Centre of the South Green Bioinformatics platform (http://www.southgreen.fr/)

The authors are thankful to Dr. Franck Molina for his key role at the beginning of this project and all the fruitful and friendly discussions

we are thankful to Peter Biggins for the careful and critical review of this manuscript.

\section{REFERENCES}

Argout, X., Salse, J., Aury, J.-M., Guiltinan, M.J., Droc, G., Gouzy, J., Allegre, M., Chaparro, C., Legavre, T., Maximova, S.N., et al. (2011). The genome of Theobroma cacao. Nat. Genet. 43, 101-108. 
Arondel, V., Vergnolle, C., Cantrel, C., and Kader, J. (2000). Lipid transfer proteins are encoded by a small multigene family in Arabidopsis thaliana. Plant Sci. Int. J. Exp. Plant Biol. 157, 1-12. Aziz, N., Paiva, N.L., May, G.D., and Dixon, R.A. (2005). Transcriptome analysis of alfalfa glandular trichomes. Planta 221, 28-38.

Bendtsen, J.D., Nielsen, H., von Heijne, G., and Brunak, S. (2004). Improved prediction of signal peptides: SignalP 3.0. J. Mol. Biol. 340, 783-795.

Benkert, P., Tosatto, S.C.E., and Schomburg, D. (2008). QMEAN: A comprehensive scoring function for model quality assessment. Proteins 71, 261-277.

Benkert, P., Biasini, M., and Schwede, T. (2011). Toward the estimation of the absolute quality of individual protein structure models. Bioinforma. Oxf. Engl. 27, 343-350.

Berman, H.M., Westbrook, J., Feng, Z., Gilliland, G., Bhat, T.N., Weissig, H., Shindyalov, I.N., and Bourne, P.E. (2000). The Protein Data Bank. Nucleic Acids Res. 28, 235-242.

Boutrot, F., Guirao, A., Alary, R., Joudrier, P., and Gautier, M.-F. (2005). Wheat non-specific lipid transfer protein genes display a complex pattern of expression in developing seeds.

Biochim. Biophys. Acta 1730, 114-125.

Boutrot, F., Chantret, N., and Gautier, M.-F. (2008). Genome-wide analysis of the rice and Arabidopsis non-specific lipid transfer protein (nsLtp) gene families and identification of wheat nsLtp genes by EST data mining. BMC Genomics 9, 86.

Bucher, M., Wegmüller, S., and Drissner, D. (2009). Chasing the structures of small molecules in arbuscular mycorrhizal signaling. Curr. Opin. Plant Biol. 12, 500-507.

Buhot, N., Douliez, J.P., Jacquemard, A., Marion, D., Tran, V., Maume, B.F., Milat, M.L., Ponchet, M., Mikès, V., Kader, J.C., et al. (2001). A lipid transfer protein binds to a receptor involved in the control of plant defence responses. FEBS Lett. 509, 27-30.

Buhot, N., Gomès, E., Milat, M.-L., Ponchet, M., Marion, D., Lequeu, J., Delrot, S., CoutosThévenot, P., and Blein, J.-P. (2004). Modulation of the biological activity of a tobacco LTP1 by lipid complexation. Mol. Biol. Cell 15, 5047-5052.

Cameron, K.D., Teece, M.A., and Smart, L.B. (2006). Increased accumulation of cuticular wax and expression of lipid transfer protein in response to periodic drying events in leaves of tree tobacco. Plant Physiol. 140, 176-183.

Cammue, B.P., Thevissen, K., Hendriks, M., Eggermont, K., Goderis, I.J., Proost, P., Van Damme, J., Osborn, R.W., Guerbette, F., and Kader, J.C. (1995). A potent antimicrobial protein from onion seeds showing sequence homology to plant lipid transfer proteins. Plant Physiol. 109, 445-455.

Catherinot, V., and Labesse, G. (2004). ViTO: tool for refinement of protein sequence-structure alignments. Bioinforma. Oxf. Engl. 20, 3694-3696.

Chae, K., and Lord, E.M. (2011). Pollen tube growth and guidance: roles of small, secreted proteins. Ann. Bot. 108, 627-636.

Chae, K., Kieslich, C.A., Morikis, D., Kim, S.-C., and Lord, E.M. (2009). A gain-of-function mutation of Arabidopsis lipid transfer protein 5 disturbs pollen tube tip growth and fertilization. Plant Cell 21, 3902-3914. 
Chanda, B., Xia, Y., Mandal, M.K., Yu, K., Sekine, K.-T., Gao, Q., Selote, D., Hu, Y., Stromberg, A., Navarre, D., et al. (2011). Glycerol-3-phosphate is a critical mobile inducer of systemic immunity in plants. Nat. Genet. 43, 421-427.

Cheng, C.-S., Chen, M.-N., Lai, Y.-T., Chen, T., Lin, K.-F., Liu, Y.-J., and Lyu, P.-C. (2008). Mutagenesis study of rice nonspecific lipid transfer protein 2 reveals residues that contribute to structure and ligand binding. Proteins 70, 695-706.

Choi, Y.E., Lim, S., Kim, H.-J., Han, J.Y., Lee, M.-H., Yang, Y., Kim, J.-A., and Kim, Y.-S. (2012). Tobacco NtLTP1, a glandular-specific lipid transfer protein, is required for lipid secretion from glandular trichomes. Plant J. Cell Mol. Biol. 70, 480-491.

Clark, A.M., and Bohnert, H.J. (1999). Cell-specific expression of genes of the lipid transfer protein family from Arabidopsis thaliana. Plant Cell Physiol. 40, 69-76.

Cong, Q., and Grishin, N.V. (2012). MESSA: MEta-Server for protein Sequence Analysis. BMC Biol. 10, 82 .

Debono, A., Yeats, T.H., Rose, J.K.C., Bird, D., Jetter, R., Kunst, L., and Samuels, L. (2009). Arabidopsis LTPG is a glycosylphosphatidylinositol-anchored lipid transfer protein required for export of lipids to the plant surface. Plant Cell 21, 1230-1238.

Desper, R., and Gascuel, O. (2002). Fast and accurate phylogeny reconstruction algorithms based on the minimum-evolution principle. J. Comput. Biol. J. Comput. Mol. Cell Biol. 9, 687-705. Douliez, J.-P., Michon, T., Elmorjani, K., \& Marion, D. (2000). Mini Review: Structure, Biological and Technological Functions of Lipid Transfer Proteins and Indolines, the Major Lipid Binding Proteins from Cereal Kernels. Journal of Cereal Science, 32(1), 1-20. Douliez, J.P., Pato, C., Rabesona, H., Mollé, D., and Marion, D. (2001b). Disulfide bond assignment, lipid transfer activity and secondary structure of a 7-kDa plant lipid transfer protein, LTP2. Eur. J. Biochem. 268, 1400-1403.

Douliez, J.P., Jégou, S., Pato, C., Mollé, D., Tran, V., and Marion, D. (2001a). Binding of two mono-acylated lipid monomers by the barley lipid transfer protein, LTP1, as viewed by fluorescence, isothermal titration calorimetry and molecular modelling. Eur. J. Biochem. 268, 384-388.

Dufayard, J.-F., Duret, L., Penel, S., Gouy, M., Rechenmann, F., and Perrière, G. (2005). Tree pattern matching in phylogenetic trees: automatic search for orthologs or paralogs in homologous gene sequence databases. Bioinforma. Oxf. Engl. 21, 2596-2603.

Edgar, R.C. (2004). MUSCLE: a multiple sequence alignment method with reduced time and space complexity. BMC Bioinformatics 5, 113.

Edstam, M.M., Viitanen, L., Salminen, T.A., and Edqvist, J. (2011). Evolutionary history of the non-specific lipid transfer proteins. Mol. Plant 4, 947-964.

Erdin, S., Ward, R.M., Venner, E., and Lichtarge, O. (2010). Evolutionary trace annotation of protein function in the structural proteome. J. Mol. Biol. 396, 1451-1473.

Gao, M., Yang, F., Zhang, L., Su, Z., and Huang, Y. (2017). Exploring the sequence-structurefunction relationship for the intrinsically disordered $\beta \gamma$-crystallin Hahellin. J. Biomol. Struct. Dyn. 1-11. 
Ge, X., Chen, J., Li, N., Lin, Y., Sun, C., and Cao, K. (2003). Resistance function of rice lipid transfer protein LTP110. J. Biochem. Mol. Biol. 36, 603-607.

Girault, T., François, J., Rogniaux, H., Pascal, S., Delrot, S., Coutos-Thévenot, P., and Gomès, E. (2008). Exogenous application of a lipid transfer protein-jasmonic acid complex induces protection of grapevine towards infection by Botrytis cinerea. Plant Physiol. Biochem. PPB 46, $140-149$.

Guindon, S., Dufayard, J.-F., Lefort, V., Anisimova, M., Hordijk, W., and Gascuel, O. (2010). New algorithms and methods to estimate maximum-likelihood phylogenies: assessing the performance of PhyML 3.0. Syst. Biol. 59, 307-321.

Henikoff, S., and Henikoff, J.G. (1994). Position-based sequence weights. J. Mol. Biol. 243, 574-578.

Hoh, F., Pons, J.-L., Gautier, M.-F., de Lamotte, F., and Dumas, C. (2005). Structure of a liganded Type-2 non-specific lipid-transfer protein from wheat and the molecular basis of lipid binding. Acta Crystallogr. D Biol. Crystallogr. 61, 397-406.

Huson, D.H., and Scornavacca, C. (2012). Dendroscope 3: an interactive tool for rooted phylogenetic trees and networks. Syst. Biol. 61, 1061-1067.

Jang, C.S., Jung, J.H., Yim, W.C., Lee, B.-M., Seo, Y.W., and Kim, W. (2007). Divergence of genes encoding non-specific lipid transfer proteins in the poaceae family. Mol. Cells 24, 215223.

José-Estanyol, M., Gomis-Rüth, F.X., and Puigdomènech, P. (2004). The eight-cysteine motif, a versatile structure in plant proteins. Plant Physiol. Biochem. PPB 42, 355-365.

Kader, J.-C. (1996). LIPID-TRANSFER PROTEINS IN PLANTS. Annu. Rev. Plant Physiol. Plant Mol. Biol. 47, 627-654.

Kader, J.C., Julienne, M., and Vergnolle, C. (1984). Purification and characterization of a spinach-leaf protein capable of transferring phospholipids from liposomes to mitochondria or chloroplasts. Eur. J. Biochem. 139, 411-416.

Katoh, K., and Toh, H. (2010). Parallelization of the MAFFT multiple sequence alignment program. Bioinforma. Oxf. Engl. 26, 1899-1900.

Katoh, K., Misawa, K., Kuma, K., and Miyata, T. (2002). MAFFT: a novel method for rapid multiple sequence alignment based on fast Fourier transform. Nucleic Acids Res. 30, 3059-3066. Konagurthu, A.S., Whisstock, J.C., Stuckey, P.J., and Lesk, A.M. (2006). MUSTANG: a multiple structural alignment algorithm. Proteins 64, 559-574.

Kurowski, M.A., and Bujnicki, J.M. (2003). GeneSilico protein structure prediction meta-server. Nucleic Acids Res. 31, 3305-3307.

Lange, B.M., Wildung, M.R., Stauber, E.J., Sanchez, C., Pouchnik, D., and Croteau, R. (2000). Probing essential oil biosynthesis and secretion by functional evaluation of expressed sequence tags from mint glandular trichomes. Proc. Natl. Acad. Sci. U. S. A. 97, 2934-2939.

Li, X., Gasic, K., Cammue, B., Broekaert, W., and Korban, S.S. (2003). Transgenic rose lines harboring an antimicrobial protein gene, Ace-AMP1, demonstrate enhanced resistance to powdery mildew ( Sphaerotheca pannosa). Planta 218, 226-232. 
Lichtarge, O., Bourne, H.R., and Cohen, F.E. (1996). An evolutionary trace method defines binding surfaces common to protein families. J. Mol. Biol. 257, 342-358.

Liu, W., Huang, D., Liu, K., Hu, S., Yu, J., Gao, G., and Song, S. (2010). Discovery, identification and comparative analysis of non-specific lipid transfer protein (nsLtp) family in Solanaceae. Genomics Proteomics Bioinformatics 8, 229-237.

van Loon, L.C., Rep, M., and Pieterse, C.M.J. (2006). Significance of inducible defense-related proteins in infected plants. Annu. Rev. Phytopathol. 44, 135-162.

Maghuly, F., Borroto-Fernandez, E.G., Khan, M.A., Herndl, A., Marzban, G., and Laimer, M. (2009). Expression of calmodulin and lipid transfer protein genes in Prunus incisa x serrula under different stress conditions. Tree Physiol. 29, 437-444.

Maldonado, A.M., Doerner, P., Dixon, R.A., Lamb, C.J., and Cameron, R.K. (2002). A putative lipid transfer protein involved in systemic resistance signalling in Arabidopsis. Nature 419, 399403.

Molina, A., and García-Olmedo, F. (1993). Developmental and pathogen-induced expression of three barley genes encoding lipid transfer proteins. Plant J. Cell Mol. Biol. 4, 983-991.

Mollet, J.C., Park, S.Y., Nothnagel, E.A., and Lord, E.M. (2000). A lily stylar pectin is necessary for pollen tube adhesion to an in vitro stylar matrix. Plant Cell 12, 1737-1750.

Murzin, A.G., Brenner, S.E., Hubbard, T., and Chothia, C. (1995). SCOP: a structural classification of proteins database for the investigation of sequences and structures. J. Mol. Biol. 247, 536-540.

Nieuwland, J., Feron, R., Huisman, B.A.H., Fasolino, A., Hilbers, C.W., Derksen, J., and Mariani, C. (2005). Lipid transfer proteins enhance cell wall extension in tobacco. Plant Cell 17, 2009-2019.

Orengo, C.A., Michie, A.D., Jones, S., Jones, D.T., Swindells, M.B., and Thornton, J.M. (1997). CATH--a hierarchic classification of protein domain structures. Struct. Lond. Engl. 19935 , 1093-1108.

Ortiz, A.R., Strauss, C.E.M., and Olmea, O. (2002). MAMMOTH (matching molecular models obtained from theory): an automated method for model comparison. Protein Sci. Publ. Protein Soc. 11, 2606-2621.

Ovchinnikov, S., Park, H., Varghese, N., Huang, P.-S., Pavlopoulos, G.A., Kim, D.E., Kamisetty, H., Kyrpides, N.C., and Baker, D. (2017). Protein structure determination using metagenome sequence data. Science 355, 294-298.

Liu F, Zhang X, Lu C, Zeng X, Li Y, Fu D, Wu G. Non-specific lipid transfer proteins in plants: presenting new advances and an integrated functional analysis. J Exp. Bot. (2015)

Pacios, L.F., Gómez-Casado, C., Tordesillas, L., Palacín, A., Sánchez-Monge, R., and DíazPerales, A. (2012). Computational study of ligand binding in lipid transfer proteins: Structures, interfaces, and free energies of protein-lipid complexes. J. Comput. Chem. 33, 1831-1844. Park, S.Y., Jauh, G.Y., Mollet, J.C., Eckard, K.J., Nothnagel, E.A., Walling, L.L., and Lord, E.M. (2000). A lipid transfer-like protein is necessary for lily pollen tube adhesion to an in vitro stylar matrix. Plant Cell 12, 151-164. 
Pii, Y., Astegno, A., Peroni, E., Zaccardelli, M., Pandolfini, T., and Crimi, M. (2009). The Medicago truncatula N5 gene encoding a root-specific lipid transfer protein is required for the symbiotic interaction with Sinorhizobium meliloti. Mol. Plant-Microbe Interact. MPMI 22, 1577-1587.

Pii, Y., Molesini, B., and Pandolfini, T. (2013). The involvement of Medicago truncatula nonspecific lipid transfer protein N5 in the control of rhizobial infection. Plant Signal. Behav. 8, e24836.

Pons, J.-L., and Labesse, G. (2009). @TOME-2: a new pipeline for comparative modeling of protein-ligand complexes. Nucleic Acids Res. 37, W485-491.

Poznanski, J., Sodano, P., Suh, S.W., Lee, J.Y., Ptak, M., and Vovelle, F. (1999). Solution structure of a lipid transfer protein extracted from rice seeds. Comparison with homologous proteins. Eur. J. Biochem. 259, 692-708.

Pyee, J., and Kolattukudy, P.E. (1995). The gene for the major cuticular wax-associated protein and three homologous genes from broccoli (Brassica oleracea) and their expression patterns. Plant J. Cell Mol. Biol. 7, 49-59.

Pyee, J., Yu, H., and Kolattukudy, P.E. (1994). Identification of a lipid transfer protein as the major protein in the surface wax of broccoli (Brassica oleracea) leaves. Arch. Biochem. Biophys. 311, 460-468.

Qin, X., Liu, Y., Mao, S., Li, T., Wu, H., Chu, C., and Wang, Y. (2011). Genetic transformation of lipid transfer protein encoding gene in Phalaenopsis amabilis to enhance cold resistance.

Euphytica 177, 33-43.

Radauer, C., and Breiteneder, H. (2007). Evolutionary biology of plant food allergens. J. Allergy Clin. Immunol. 120, 518-525.

Radauer, C., Bublin, M., Wagner, S., Mari, A., and Breiteneder, H. (2008). Allergens are distributed into few protein families and possess a restricted number of biochemical functions. J. Allergy Clin. Immunol. 121, 847-852.e7.

Rocklin, G.J., Chidyausiku, T.M., Goreshnik, I., Ford, A., Houliston, S., Lemak, A., Carter, L., Ravichandran, R., Mulligan, V.K., Chevalier, A., et al. (2017). Global analysis of protein folding using massively parallel design, synthesis, and testing. Science 357, 168-175.

Salcedo, G., Sánchez-Monge, R., Barber, D., and Díaz-Perales, A. (2007). Plant non-specific lipid transfer proteins: an interface between plant defence and human allergy. Biochim. Biophys. Acta 1771, 781-791.

Sawano, Y., Hatano, K., Miyakawa, T., Komagata, H., Miyauchi, Y., Yamazaki, H., and Tanokura, M. (2008). Proteinase inhibitor from ginkgo seeds is a member of the plant nonspecific lipid transfer protein gene family. Plant Physiol. 146, 1909-1919.

Silverstein, K.A.T., Moskal, W.A., Wu, H.C., Underwood, B.A., Graham, M.A., Town, C.D., and VandenBosch, K.A. (2007). Small cysteine-rich peptides resembling antimicrobial peptides have been under-predicted in plants. Plant J. Cell Mol. Biol. 51, 262-280. 
Sror, H.A.M., Tischendorf, G., Sieg, F., Schmitt, J.M., and Hincha, D.K. (2003). Cryoprotectin protects thylakoids during a freeze-thaw cycle by a mechanism involving stable membrane binding. Cryobiology 47, 191-203.

Sterk, P., Booij, H., Schellekens, G.A., Van Kammen, A., and De Vries, S.C. (1991). Cellspecific expression of the carrot EP2 lipid transfer protein gene. Plant Cell 3, 907-921.

Sun, J.-Y., Gaudet, D.A., Lu, Z.-X., Frick, M., Puchalski, B., and Laroche, A. (2008). Characterization and antifungal properties of wheat nonspecific lipid transfer proteins. Mol. Plant-Microbe Interact. MPMI 21, 346-360.

Thoma, S., Kaneko, Y., and Somerville, C. (1993). A non-specific lipid transfer protein from Arabidopsis is a cell wall protein. Plant J. Cell Mol. Biol. 3, 427-436.

Trevino, M.B., and OConnell, M.A. (1998). Three drought-responsive members of the nonspecific lipid-transfer protein gene family in Lycopersicon pennellii show different developmental patterns of expression. Plant Physiol. 116, 1461-1468.

Wang, H.W., Hwang, S.-G., Karuppanapandian, T., Liu, A., Kim, W., and Jang, C.S. (2012a). Insight into the molecular evolution of non-specific lipid transfer proteins via comparative analysis between rice and sorghum. DNA Res. Int. J. Rapid Publ. Rep. Genes Genomes 19, 179194.

Wang, N.-J., Lee, C.-C., Cheng, C.-S., Lo, W.-C., Yang, Y.-F., Chen, M.-N., and Lyu, P.-C. (2012b). Construction and analysis of a plant non-specific lipid transfer protein database (nsLTPDB). BMC Genomics 13 Suppl 1, S9. 


\section{Figure $\mathbf{1}$ (on next page)}

Effect of alternate cysteine residue alignments on the superposition of type I and II nsLTP experimentally determined structures.

(A1) Common alignment of Cys5 (type I), Cys5' (types II and IV) (green) and Cys6 (type I), Cys6' (types II and IV) (magenta) of nsLTP sequences generated by MUSCLE. Only nsLTPS (PDB IDS) with known experimental structures were considered.(A2) 3D projection of this alignment leads to a RMSD of $7.32 \AA$ between type I (blue backbone) Cys6 and type II (pink backbone) Cys6', colorized as in (A1).(B1) Type I, II and IV nsLTP alignment generated by the MAFFT program, suggesting that type I Cys5 (dark green) corresponds to type II Cys6' (light green) rather than type II Cys5'.(B2) 3D projection of this alignment leads to a RMSD of 2.15 $\AA$ between type I Cys5 and type II Cys6', colorized as in (B1). Note that type IV nsLTPs are structurally close to type II nSLTPS. 


\begin{abstract}
$\begin{array}{llllllll}\text { A1 } & \text { C1 } & \text { C2 } & \text { C3 C4 } & \text { C5 C6 } & \text { C7 }\end{array}$
-IDCGHVDSLRPCLSYVQG-GP-GPSGQCCDGVKNLHNQARSQSDROSACNCLKGIARGIH--NLNEDNARSIPPKCGV-NLPYTISLNIDCSRV- 1BWO TyPe I ITCGOVNSAVGPCITYARG-GA-GPSAACCSGVRSLKAAASTTADRRTACNCLKNAARGIK--GLNAGNAASTPSKCGV-SVPYTISASIDCSRVS 1RZL TYPe I

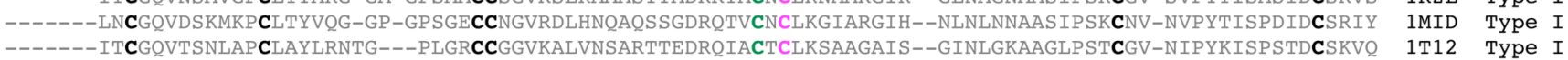
------MTCGQVQGNLAQCIGFLQKGGV-VPPS-CCTGVKNILNSSRTTADRRAVCSCLKAAAGAVR--GINPNNAEALPGKCGV-NIPYKISTSTNCNSIN 1 SIY TYPE I ------ITCGOVSSALAPCIPYVRGGGA-VPPA-CCNGIRNVNNLARTTPDROAACNCLKOLSASVP--GVNPNNAAALPGKCGV-HIPYKISASTNCATVK 2ALG TyPe I ------AISCGOVASAIAPCISYARGOGS-GPSAGCCSGVRSLNNAARTTADRRAACNCLKNAAAGVS--GLNAGNAASIPSKCGV-SIPYTISTSTDCSRVN 1FK5 TYPe I
\end{abstract}

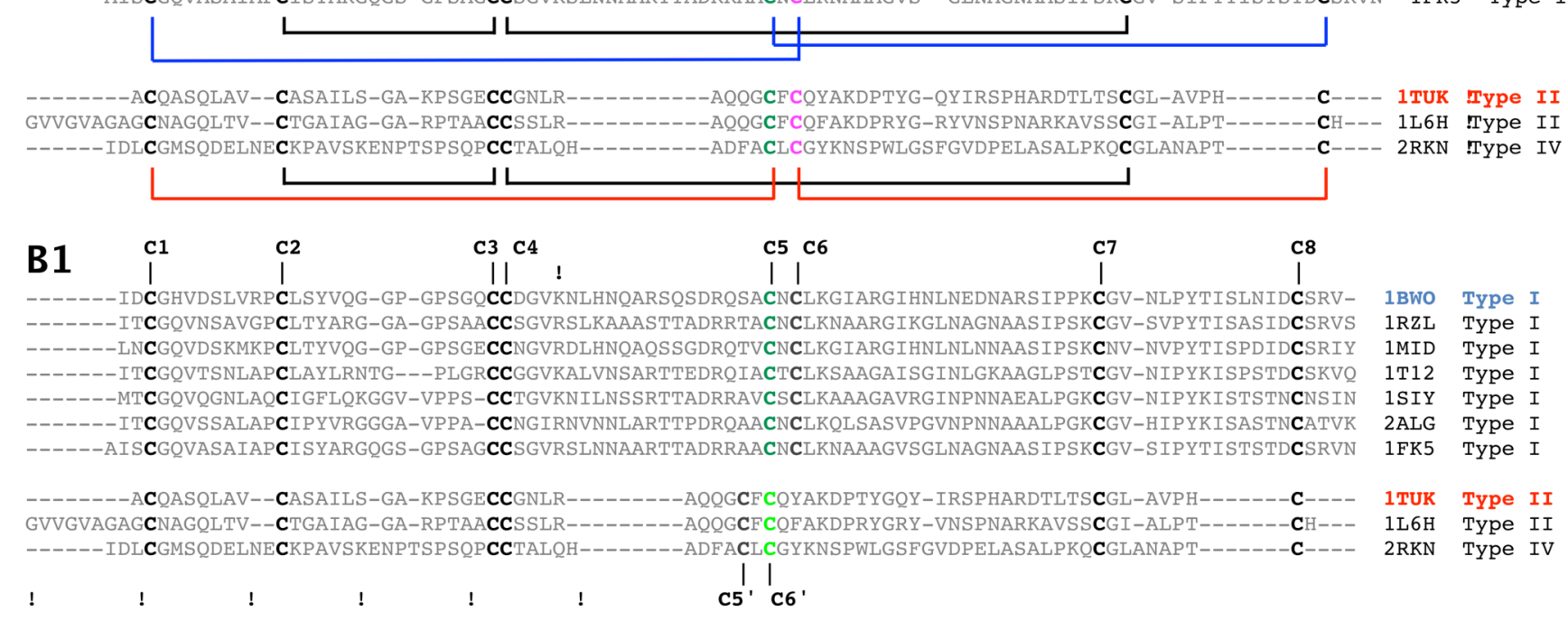

A2

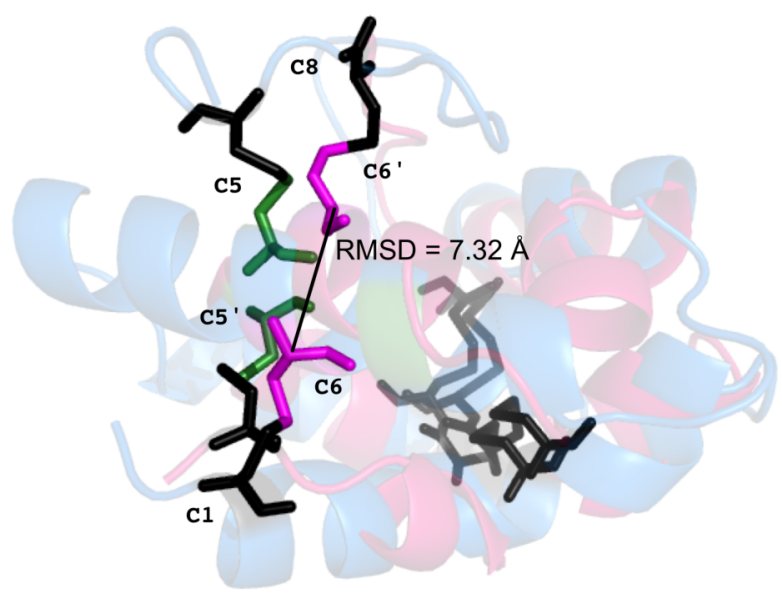

B2

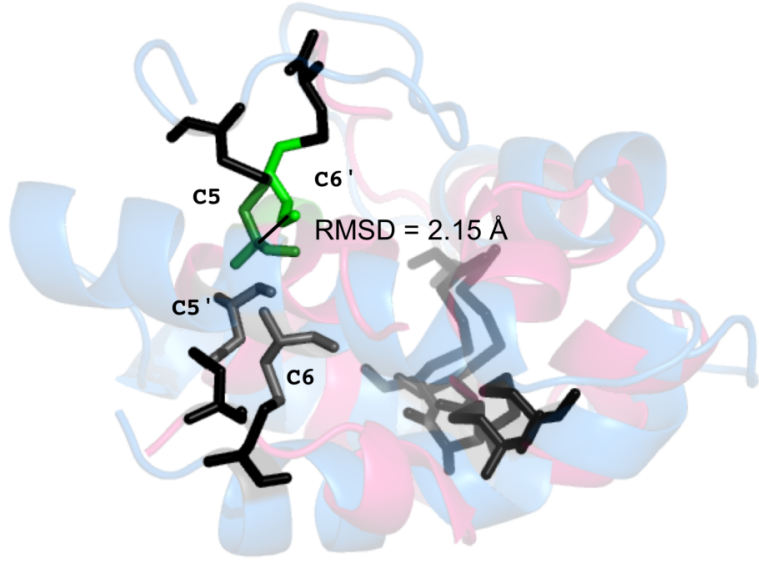


Figure 2 (on next page)

NsLTP sequence classification.

Dendrogram built on MAFFT alignment of the 797 nsLTP sequences, using Dendroscope program (Huson and Scornavacca 2012). The different nsLTP types are displayed using various colors and the number of sequences in each type is specified in parenthesis. Branch support values of each group are indicated on the corresponding nodes. 


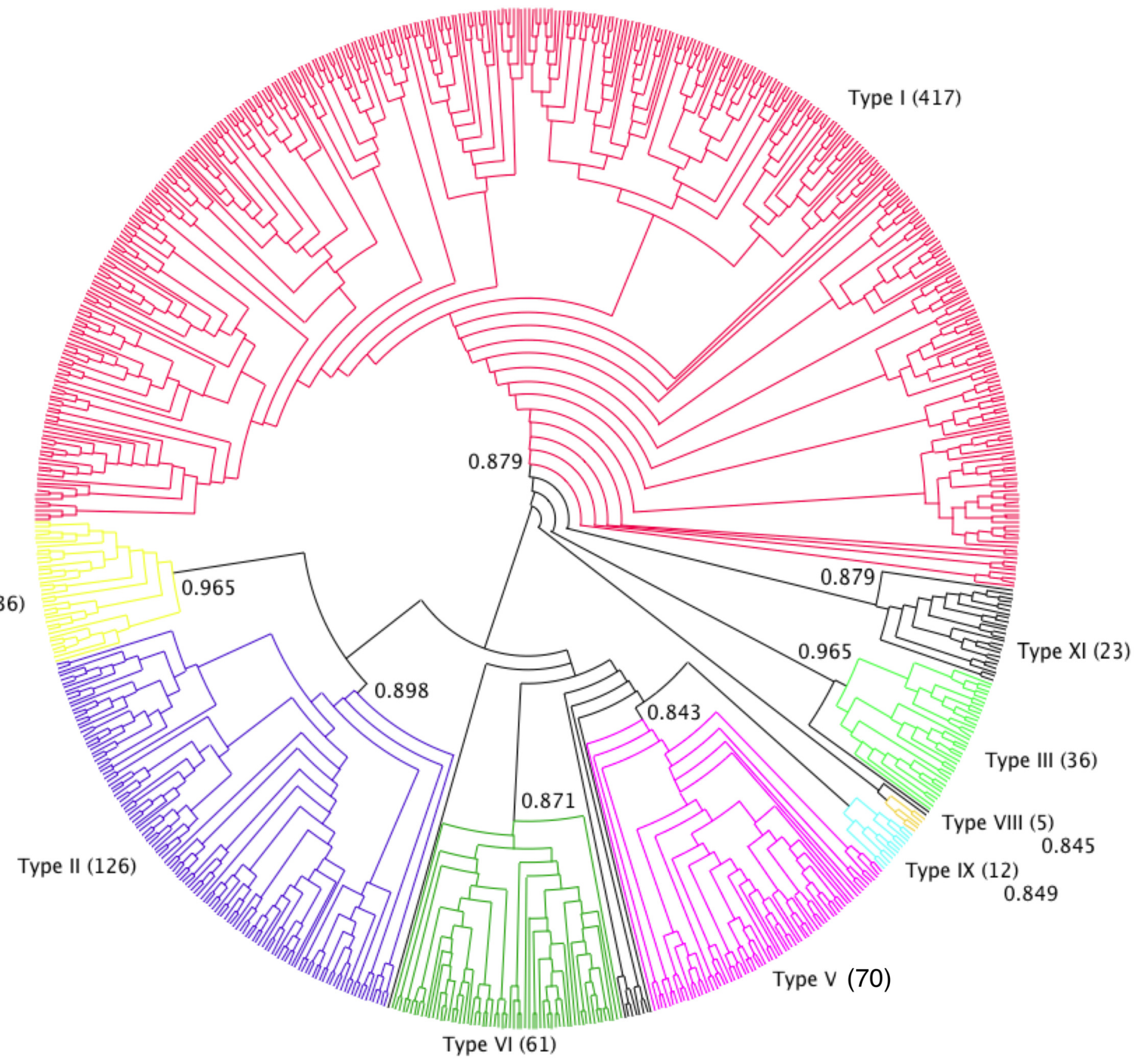


Figure 3 (on next page)

Consensus sequence alignment for all nsLTP types.

The indicated amino acids are the most frequent for each type of nSLTP. Black residues are strongly conserved over all nSLTP while colored residues are specifically conserved in a few types of nSLTP only (coloring method explained in the appendix file). Vertical arrows indicate residues analyzed in detail in the text below. 


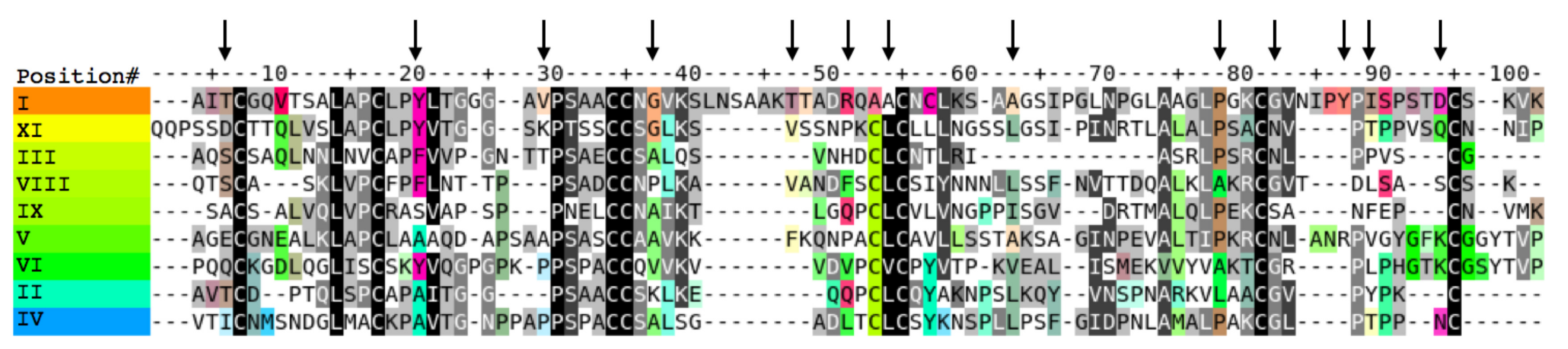


Figure 4 (on next page)

Cartoon representation of the crystallographic structures Imid (type I), 1tuk (type II) and 2rkn (type IV).

The residues are numbered and colored as in the multiple sequence alignment of $J 1$. The ligands are represented as ball and sticks (carbon in white, oxygen in red). Some determining amino acid side chains are also displayed. 

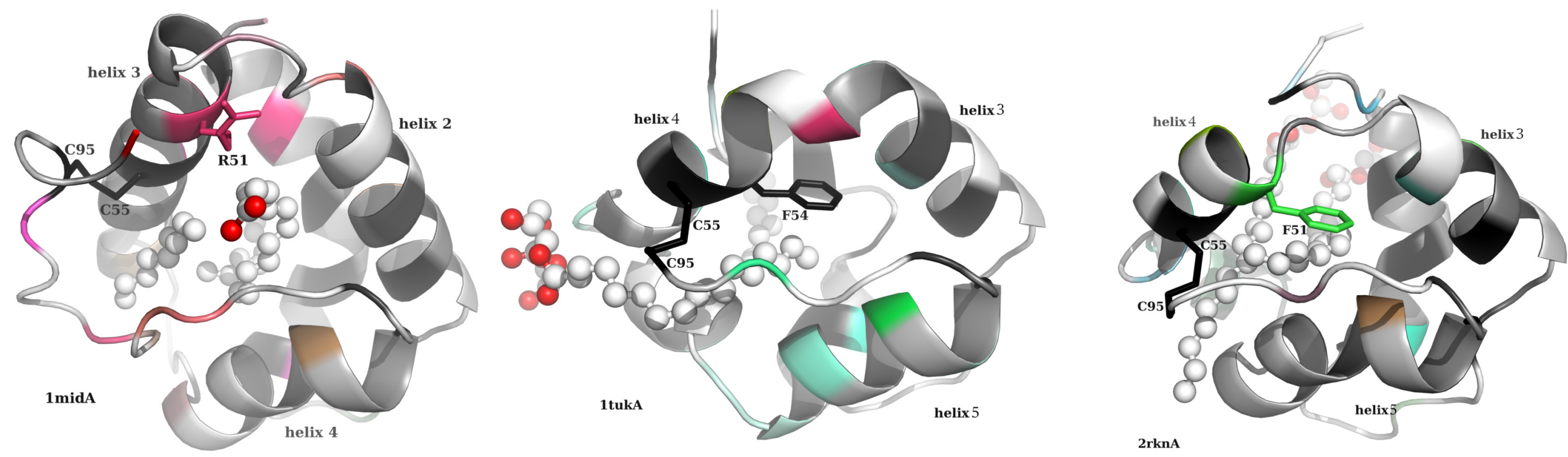
Figure $\mathbf{5}$ (on next page)

NsLTP structure classification.

Dendrogram built on Mustang structure-based sequence alignment of the 727 nsLTPs for which a reliable 3D model has been calculated. The two main fold types are displayed in red (type 1 fold) and black (type 12 fold). In order to study their distribution in term of structural families, nSLTP structures are colored according to the previously determined phylogenetic type they belong to (same colors as used in fig.2). Phylogenetic type I nsLTPs display the type 1 fold and all other nsLTPs follow the type 2 fold. 


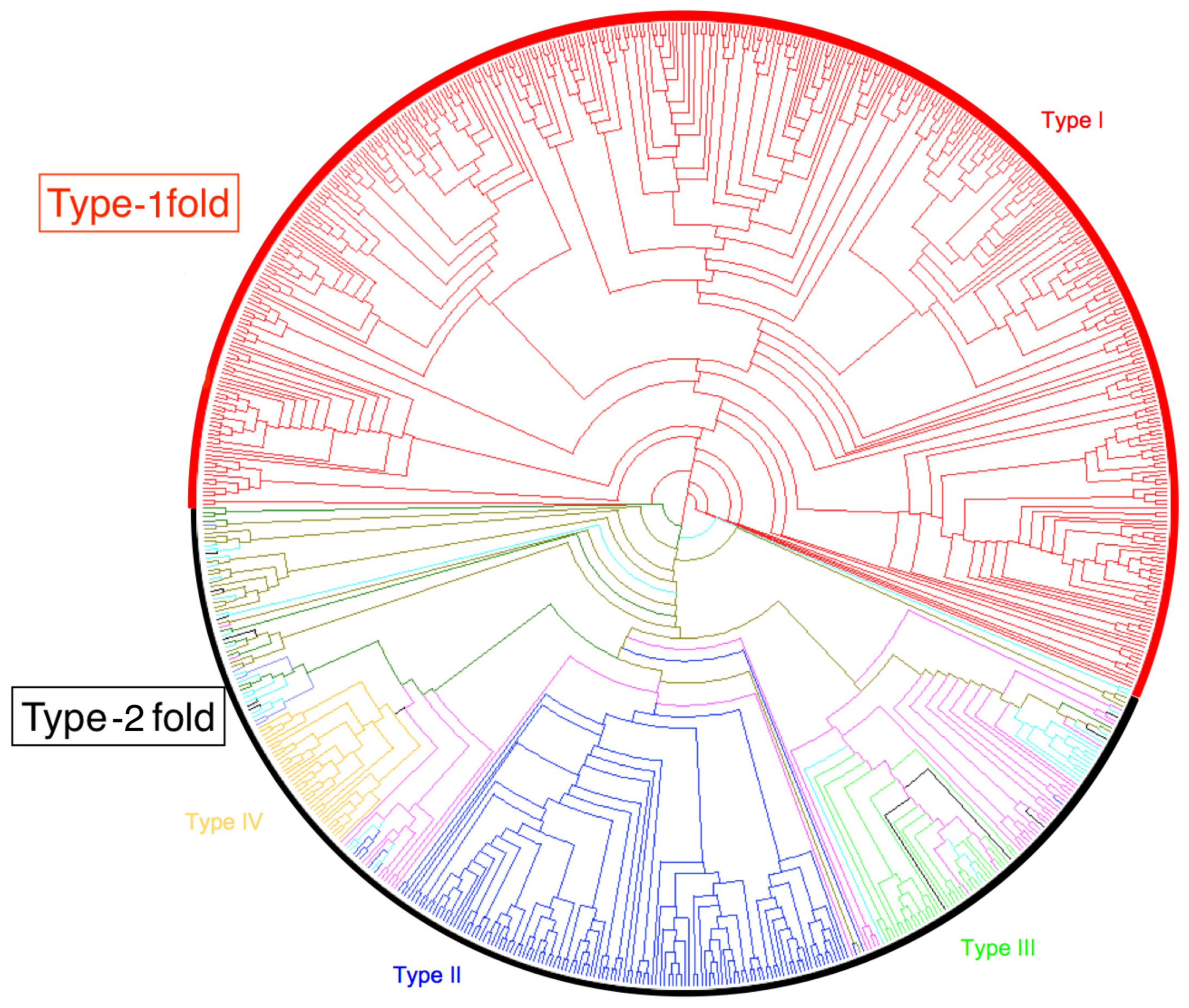




\section{Figure 6 (on next page)}

InTreeGreat view of the structure tree.

The left pane shows the phylogenic tree of the nsLTP structures colored according to type and the right pane represents a close-up of the Type I (colored in red) part of the tree. For clarity, some sub tree parts for which no annotation was available have been collapsed. They appear as grey triangle and the number of structures they contain is indicated.NsLTPs for which a functional annotation is available are highlighted with a grey box in the left column. On the right side of the tree several columns appear that correspond to annotations (PO, GO), number of leaves in a collapsed sub-tree together with colored boxes. The first column of boxes shows alternative colors to enhance the clusters, the other ones correspond to each keyword selected among the annotations of the database (here: "defense" or "resistance"). Keywords "defense" or "resistance" used in functional annotation are highlighted with a colored box (blue and red respectively). The "defense cluster" (see next paragraph) has been enlarged (black border) for a better view. 
Figure 7 (on next page)

Conserved amino acid residues among the so-called defense cluster, on the $3 D$ structure of nsLTP 525, ("LTP", UniProtKB - Q1KMV1).

The more the residue is conserved in the 3D alignment, the redder its colour appears, thenorange, yellow and green. Residues with no significative conservation appears in white on the figure. Residues highlighted in table $X$ and which potential functional implication is discussed (see text) are labeled on the figure. 


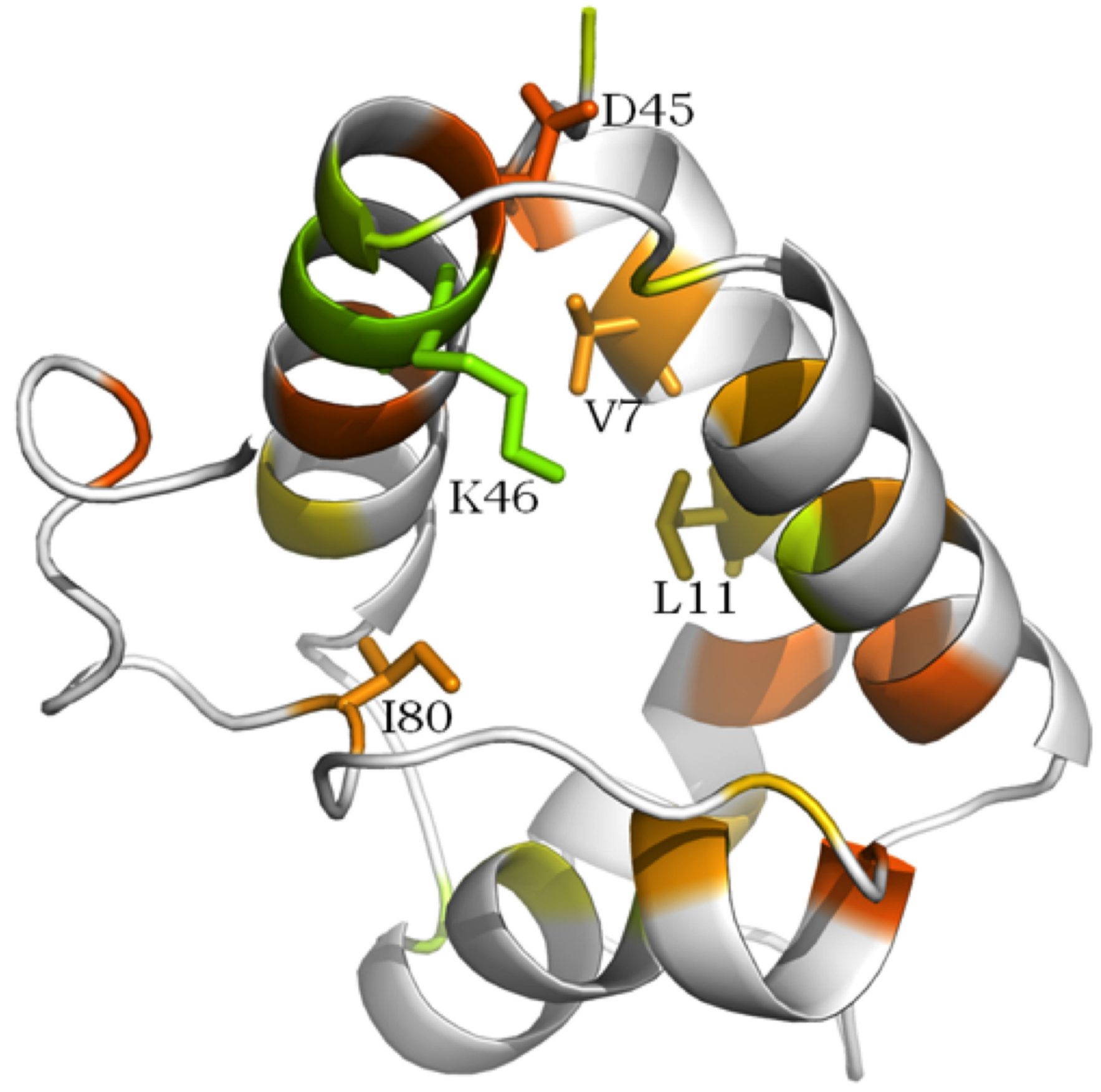




\section{Table $\mathbf{1}$ (on next page)}

Qmean scores obtained by the 797 theoretical models of nsLTPs of this study.

Models obtained by @tome2 present an overall good quality as shown in Table 1 that summarizes the Qmean scores. For $95,85 \%$ of the models, Qmean scores are above 0.4 and $57 \%$ of the models obtained scores ranging from 0.5 to 0.9 , which correspond to scores for high-resolution proteins. It is known that disordered protein regions are very flexible regions. While submitted to automatic evaluation, these flexible regions will be considered as regions of bad quality modeling, leading to lower Qmean scores (Benkert, Tosatto et al. 2008;

Benkert, Biasini et al. 2011). Small proteins tend to have lower scores than larger proteins, because of the lower proportion of secondary structures compared to random coils. However, the set of theoretical models calculated by @tome2 obtained overall good Qmean scores.NB: for 121 theoretical structures, the polypeptide chain could not be fully built and the resulting models were lacking at least one of the 8 cysteine residues. Such models were discarded and a new pool of 677 structures was retained for further analysis. The models are available at: http://atome.cbs.cnrs.fr/AT2B/SERVER/LTP.html 
1

\begin{tabular}{|c|c|c|}
\hline Qmean score (Q) & Nb. of models & Dataset proportion \\
\hline $\mathrm{Q}<0.2$ & 2 & $0.3 \%$ \\
\hline $0.2<\mathrm{Q}<0.3$ & 16 & $2 \%$ \\
\hline $0.3<\mathrm{Q}<0.4$ & 105 & $13.2 \%$ \\
\hline $0.4<\mathrm{Q}<0.5$ & 216 & $27.1 \%$ \\
\hline $0.5<\mathrm{Q}<0.6$ & 291 & $36.6 \%$ \\
\hline $0.6<\mathrm{Q}<0.7$ & 142 & $17.8 \%$ \\
\hline $0.7<\mathrm{Q}<0.8$ & 21 & $2.6 \%$ \\
\hline $0.8<\mathrm{Q}<0.9$ & 3 & $0.4 \%$ \\
\hline Total & 797 & $100 \%$ \\
\hline
\end{tabular}

2 


\section{Table 2 (on next page)}

Compared analysis of Evolutionary Trace of three groups of nsLTPS.

Compared analysis of Evolutionary Trace of three groups of nsLTPs: the defense cluster (43 proteins), the cluster containing all type 1 fold nsLTPs (402 proteins) and a group composed by all type 1 fold defense/resistance nsLTPs, including those which do not belong to the defense cluster (28 proteins). This table lists the 30\% top-ranked (= most conserved) residues identified in the defense cluster trace and shows by comparison the ranking of these same residues in the other two traces, together with their coverage, variability and rVET score. Residue positions in the reference proteins and in the structure-based sequence alignment are also indicated. Alignment position is the same in all three groups because all three alignments used to perform the traces are extracted from the general multiple alignment of all 797 nsLTPs of the study. Five residues are highlighted for they are differently conserved in the three clusters of proteins (see text). 


\begin{tabular}{|c|c|c|c|c|c|c|}
\hline \multicolumn{7}{|c|}{ Defense cluster (ref. prot. $=525$ ) } \\
\hline Rank & $\begin{array}{l}\text { Residue } \\
\text { Number }\end{array}$ & \begin{tabular}{|c|} 
Alignment \\
Position
\end{tabular} & Residue & Coverage & Variability & $\begin{array}{l}\text { rvET } \\
\text { score }\end{array}$ \\
\hline 1 & 4 & 93 & $\mathrm{C}$ & 0.10000 & C & 1.00 \\
\hline 1 & 14 & 159 & C & 0.10000 & C & 1.00 \\
\hline 1 & 29 & 228 & C & 0.10000 & $\mathrm{C}$ & 1.00 \\
\hline 1 & 30 & 229 & C & 0.10000 & C & 1.00 \\
\hline 1 & 45 & 259 & D & 0.10000 & $\mathbf{D}$ & 1.00 \\
\hline 1 & 50 & 275 & $\mathrm{C}$ & 0.10000 & $\mathrm{C}$ & 1.00 \\
\hline 1 & 52 & 277 & C & 0.10000 & C & 1.00 \\
\hline 1 & 72 & 372 & C & 0.10000 & C & 1.00 \\
\hline 1 & 86 & 432 & C & 0.10000 & C & 1.00 \\
\hline 10 & 7 & 137 & $\mathbf{V}$ & 0.13333 & AV & 1.11 \\
\hline 11 & 32 & 231 & G & 0.13333 & SG & 1.11 \\
\hline 12 & 80 & 402 & I & 0.13333 & VI & 1.11 \\
\hline 13 & 69 & 367 & $P$ & 0.14444 & PA & 1.17 \\
\hline 14 & 36 & 236 & $\mathrm{~L}$ & 0.15556 & LV & 1.28 \\
\hline 15 & 17 & 165 & $Y$ & 0.16667 & $\mathrm{FY}$ & 1.59 \\
\hline 16 & 74 & 374 & V & 0.17778 & LVIA & 1.75 \\
\hline 17 & 11 & 154 & $\mathbf{L}$ & 0.18889 & LV & 1.83 \\
\hline 18 & 54 & 289 & $\mathrm{~K}$ & 0.20000 & VKQ & 1.93 \\
\hline 19 & 65 & 360 & A & 0.21111 & TALV & 2.01 \\
\hline 20 & 40 & 247 & A & 0.22222 & TAV & 2.13 \\
\hline 21 & 1 & 63 & A & 0.23333 & AD & 2.15 \\
\hline 22 & 33 & 232 & V & 0.24444 & AVI & 2.29 \\
\hline 23 & 68 & 364 & I & 0.25556 & LI & 2.50 \\
\hline 24 & 43 & 256 & $\mathrm{~T}$ & 0.26667 & TPMAS & 2.61 \\
\hline 25 & 61 & 344 & $\mathrm{~N}$ & 0.27778 & KNSV & 2.65 \\
\hline 26 & 47 & 268 & Q & 0.28889 & RQK & 2.71 \\
\hline 27 & 46 & 266 & $\mathbf{K}$ & 0.30000 & RK & 2.75 \\
\hline
\end{tabular}

\begin{tabular}{|c|c|c|c|c|ll|c|}
\hline \multicolumn{7}{|c|}{ Fold 1 nsLTPs (ref. prot. = 437) } \\
\hline Rank & $\begin{array}{c}\text { Residue } \\
\text { Number }\end{array}$ & $\begin{array}{c}\text { Alignment } \\
\text { Position }\end{array}$ & Residue & Coverage & & Variability & $\begin{array}{c}\text { rvET } \\
\text { score }\end{array}$ \\
\hline 1 & 14 & 159 & C & 0.05376 & C & 1.00 \\
1 & 29 & 228 & C & 0.05376 & C & 1.00 \\
1 & 30 & 229 & C & 0.05376 & C & 1.00 \\
1 & 50 & 275 & C & 0.05376 & C & 1.00 \\
1 & 52 & 277 & C & 0.05376 & C & 1.00 \\
6 & 75 & 372 & C & 0.06452 & CR & 1.75 \\
7 & 4 & 93 & C & 0.07527 & CA & 3.00 \\
8 & 89 & 432 & C & 0.08602 & CDN & 4.36 \\
9 & 72 & 367 & P & 0.09677 & PASLQG & 7.27 \\
10 & 46 & 266 & R & $\mathbf{0 . 1 0 7 5 3}$ & RKTAPIQD & $\mathbf{1 1 . 5 5}$ \\
$\mathbf{1 1}$ & $\mathbf{7}$ & $\mathbf{1 3 7}$ & $\mathbf{V}$ & $\mathbf{0 . 1 1 8 2 8}$ & VALISGT & $\mathbf{1 1 . 8 1}$ \\
12 & 32 & 231 & G & 0.12903 & GSAEQVHR & 13.26 \\
13 & 36 & 236 & L & 0.13978 & LVIM & 13.58 \\
14 & 77 & 374 & V & 0.15054 & VLTAINP & 13.66 \\
\hline
\end{tabular}




\begin{tabular}{|c|c|c|c|c|c|c|}
\hline 15 & 17 & 165 & Y & 0.16129 & YFAH & 13.82 \\
\hline 16 & 40 & 247 & $A$ & 0.17204 & ATSVIRPL & 14.49 \\
\hline 17 & 68 & 360 & $A$ & 0.18280 & ATVLFIM & 14.52 \\
\hline 18 & 71 & 364 & I & 0.19355 & LIVTAPFM & 14.53 \\
\hline 19 & 54 & 289 & $\mathrm{~K}$ & 0.20430 & KVQIERLMHTS & 15.40 \\
\hline 20 & 45 & 259 & D & 0.21505 & DAENITLRG.K & 15.74 \\
\hline 21 & 83 & 402 & I & 0.22581 & IVFPLTAKW & 15.92 \\
\hline 29 & 33 & 232 & V & 0.31183 & VAILSM & 21.38 \\
\hline 32 & 47 & 268 & $\mathrm{R}$ & 0.34409 & KQRVEMIYSH & 24.45 \\
\hline 34 & 11 & 154 & I & 0.36559 & VLMIFATP & 25.38 \\
\hline 42 & 64 & 344 & $\mathrm{~N}$ & 0.45161 & NGKQDASTLERVFYI & 54.16 \\
\hline 56 & 43 & 256 & $\mathrm{~T}$ & 0.60215 & TAPGRSQKDHVMI.LFY & 38.13 \\
\hline 61 & 1 & 63 & A & 0.65591 & .AHETDVPSGFQL & 39.96 \\
\hline \multicolumn{7}{|c|}{ Defense nsLTPs outside cluster (ref. prot. $=525$ ) } \\
\hline Rank & \begin{tabular}{|l|} 
Residue \\
Number
\end{tabular} & $\begin{array}{c}\text { Alignment } \\
\text { Position }\end{array}$ & Residue & Coverage & Variability & $\begin{array}{l}\text { rvET } \\
\text { score }\end{array}$ \\
\hline$\overline{1}$ & $\overline{4}$ & 93 & $\mathrm{C}$ & 0.11111 & C & 1.00 \\
\hline 1 & 14 & 159 & C & 0.11111 & $\mathrm{C}$ & 1.00 \\
\hline 1 & 29 & 228 & C & 0.11111 & C & 1.00 \\
\hline 1 & 30 & 229 & C & 0.11111 & C & 1.00 \\
\hline 1 & 50 & 275 & $\mathrm{C}$ & 0.11111 & $\mathrm{C}$ & 1.00 \\
\hline 1 & 52 & 277 & $C$ & 0.11111 & C & 1.00 \\
\hline 1 & 72 & 372 & C & 0.11111 & C & 1.00 \\
\hline 1 & 86 & 432 & C & 0.11111 & C & 1.00 \\
\hline 1 & 7 & 137 & V & 0.11111 & V & 1.00 \\
\hline 1 & 69 & 367 & $P$ & 0.11111 & $\mathrm{P}$ & 1.00 \\
\hline 11 & 45 & 259 & D & 0.13333 & DL & 1.15 \\
\hline 12 & 80 & 402 & I & 0.13333 & IW & 1.15 \\
\hline 13 & 74 & 374 & $\mathrm{~V}$ & 0.15556 & VIN & 1.39 \\
\hline 16 & 17 & 165 & $Y$ & 0.18889 & YF & 1.67 \\
\hline 17 & 36 & 236 & $\mathrm{~L}$ & 0.18889 & LI & 1.67 \\
\hline 18 & 32 & 231 & $G$ & 0.20000 & GAV & 1.76 \\
\hline 20 & 54 & 289 & $\mathrm{~K}$ & 0.22222 & KVQ & 1.93 \\
\hline 22 & 65 & 360 & A & 0.24444 & AVF & 2.04 \\
\hline 23 & 40 & 247 & $A$ & 0.25556 & ATVS & 2.05 \\
\hline 25 & 33 & 232 & V & 0.27778 & VALI & 2.59 \\
\hline 27 & 61 & 344 & $\mathrm{~N}$ & 0.30000 & NVDR & 2.88 \\
\hline 30 & 46 & 266 & K & 0.33333 & KR & 3.25 \\
\hline 31 & 11 & 154 & $\mathbf{L}$ & 0.34444 & LIVM & 3.26 \\
\hline 36 & 43 & 256 & $\bar{T}$ & 0.40000 & TPQRS & 3.72 \\
\hline 38 & 68 & 364 & I & 0.42222 & ILV & 3.95 \\
\hline 44 & 47 & 268 & Q & 0.48889 & QRK & 4.61 \\
\hline 45 & 1 & 63 & A & 0.50000 & A.QV & 4.63 \\
\hline
\end{tabular}

